\title{
BCL-W has a fundamental role in B cell survival and lymphomagenesis
}

\author{
Clare M. Adams, ${ }^{1}$ Annette S. Kim, ${ }^{2}$ Ramkrishna Mitra, ${ }^{1}$ John K. Choi, ${ }^{3}$ Jerald Z. Gong, ${ }^{4}$ and Christine M. Eischen ${ }^{1}$ \\ 'Department of Cancer Biology, Sidney Kimmel Cancer Center, Thomas Jefferson University, Philadelphia, Pennsylvania, USA. ²Department of Pathology, Brigham and Women's Hospital, Boston, \\ Massachusetts, USA. ${ }^{3}$ Department of Pathology, St. Jude Children's Research Hospital, Memphis, Tennessee, USA. ${ }^{4}$ Department of Pathology, Anatomy, and Cell Biology, \\ Thomas Jefferson University, Philadelphia, Pennsylvania, USA.
}

\begin{abstract}
Compromised apoptotic signaling is a prerequisite for tumorigenesis. The design of effective therapies for cancer treatment depends on a comprehensive understanding of the mechanisms that govern cell survival. The antiapoptotic proteins of the BCL-2 family are key regulators of cell survival and are frequently overexpressed in malignancies, leading to increased cancer cell survival. Unlike BCL-2 and BCL-XL, the closest antiapoptotic relative BCL-W is required for spermatogenesis, but was considered dispensable for all other cell types. Here, however, we have exposed a critical role for BCL-W in B cell survival and lymphomagenesis. Loss of Bcl-w conferred sensitivity to growth factor deprivation-induced B cell apoptosis. Moreover, $B c l-w$ loss profoundly delayed MYC-mediated B cell lymphoma development due to increased MYC-induced B cell apoptosis. We also determined that MYC regulates BCL-W expression through its transcriptional regulation of specific miR. BCL-W expression was highly selected for in patient samples of Burkitt lymphoma (BL), with $88.5 \%$ expressing BCL-W. BCL-W knockdown in BL cell lines induced apoptosis, and its overexpression conferred resistance to BCL-2 family-targeting BH3 mimetics. Additionally, BCL-W was overexpressed in diffuse large B cell lymphoma and correlated with decreased patient survival. Collectively, our results reveal that BCL-W profoundly contributes to B cell lymphoma, and its expression could serve as a biomarker for diagnosis and aid in the development of better targeted therapies.
\end{abstract}

\section{Introduction}

Apoptosis is a well-orchestrated process regulated by multiple genes, particularly those belonging to the BCL-2 family. The inherent ability of cells to undergo apoptosis in response to cellular stresses, including activation of oncogenes such as MYC, functions as a safeguard against tumorigenesis (1). Consequently, alterations resulting in aberrant expression of antiapoptotic BCL-2 family members and/or loss of expression of proapoptotic BCL-2 family members are well documented in most human cancers (2). The BCL-2 family has an established role in determining whether cells should live or die. However, knowledge about whether specific BCL-2 family members (other than BCL-2 itself) are dysregulated in or contribute to human malignancies and how this information can be exploited therapeutically remains incomplete.

Dysregulated MYC expression occurs in at least $70 \%$ of human malignancies and is a known driver of Burkitt lymphoma (BL) (3). The E $\mu$-MYC-Tg mouse model of MYC-induced B cell lymphomagenesis (4) has been instrumental in delineating the roles of BCL-2 family proteins in lymphoma, which has translated to many other human cancers (5). However, relative to other BCL-2 family members, little information is known about the role of the antiapoptotic protein BCL-W in cell survival and lymphomagenesis. This is likely due to early reports showing low expression of BCL-W in pri-

Conflict of interest: The authors have declared that no conflict of interest exists. Submitted: July 11, 2016; Accepted: November 22, 2016.

Reference information: J Clin Invest. 2017;127(2):635-650

https://doi.org/10.1172/JCl89486. mary mouse hematopoietic cells and to observations that $B c l$-wdeficient mice have no overt hematopoietic defects $(6,7)$. While $\mathrm{BCL}-\mathrm{W}$ is recognized for its critical function in spermatogenesis $(8,9)$, there is emerging evidence to suggest that BCL-W may also have a role in tumorigenesis. Specifically, BCL-W overexpression cooperated with MYC to accelerate the development of myeloid leukemia (10). In addition, BCL-W overexpression was observed in patient samples of gastric and colorectal adenocarcinoma (11, 12) and in several human solid tumor cell lines (13). These data indicate that BCL-W may contribute to human cancers, but which malignancies and whether BCL-W contributes to tumorigenesis and cancer cell survival remain unclear.

Here, we report a previously unknown role for BCL-W in MYC-induced apoptosis and lymphomagenesis that we believe will have significant clinical implications. By evaluating $B C L-W$ expression in human B cell lymphomas in combination with mouse modeling to directly assess the contribution of BCL-W in MYC-driven lymphomagenesis, we determined that BCL-W has a significant role in B cell lymphoma development and survival. Furthermore, we identified a mechanism by which MYC regulates BCL-W expression. Our data illuminate an unappreciated link between MYC and BCL-W that we believe significantly extends the knowledge of MYC and the role of BCL-W in tumorigenesis, both of which should aid in improving lymphoma prognostics, diagnostics, and therapeutics.

\section{Results}

Cytokine deprivation-induced apoptosis is accelerated by loss of BCL-W. BCL-W levels were reported to be low in lymphocytes and dispens- 
A
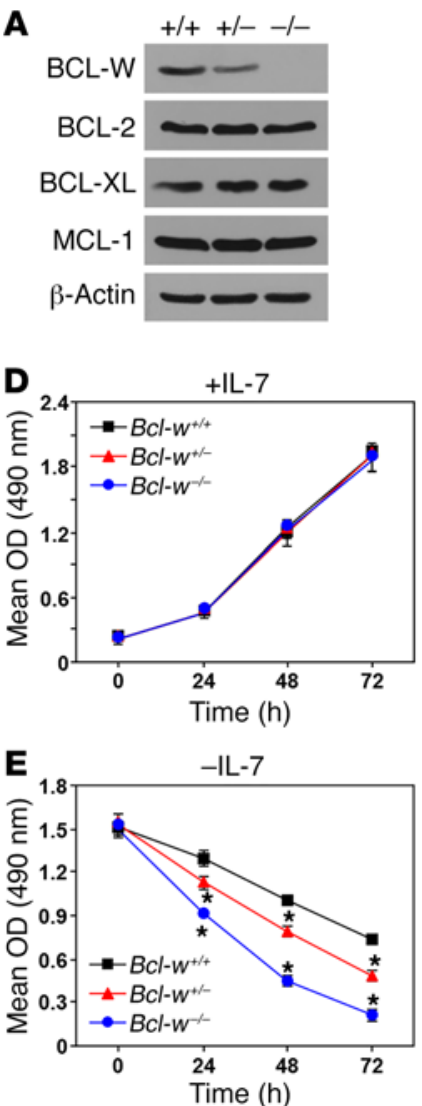

B
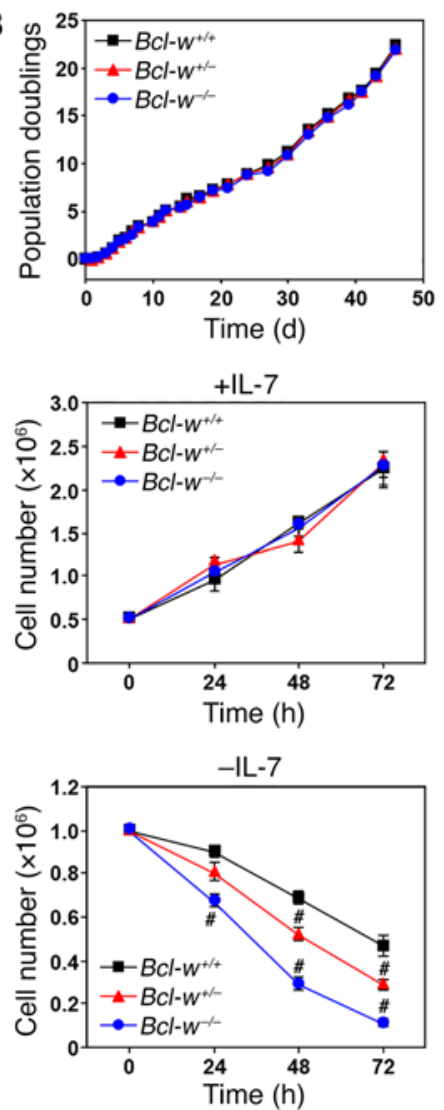
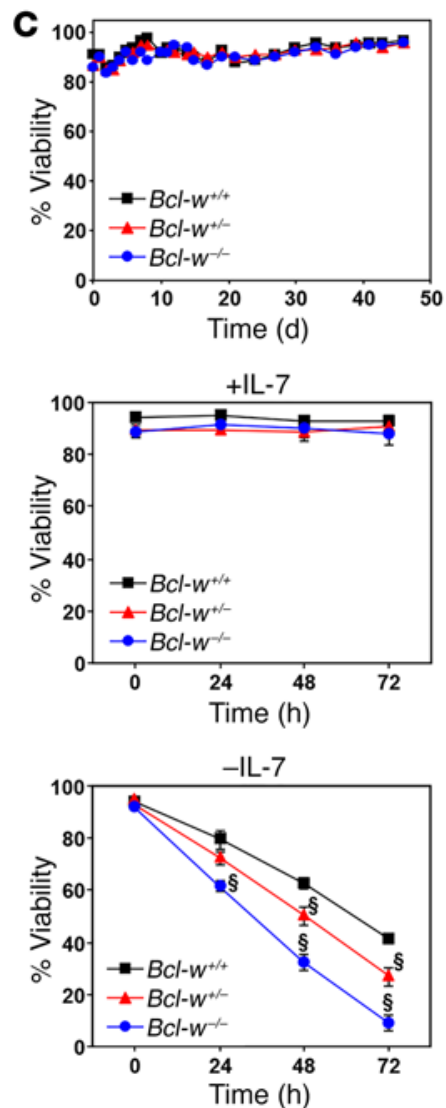

Figure 1. Cytokine deprivation-induced pre-B cell death is accelerated by loss of $\mathrm{Bcl}-\mathbf{w}$. BM from $B c \mathrm{l}-\mathrm{w}^{+/+}, \mathrm{Bcl}-\mathrm{w}^{+/-}$, and $\mathrm{Bcl}-\mathrm{w}^{-/-}$littermates was placed into IL-7-containing media on day 0 . Western blotting was performed on pre-B cells that grew out of the cultures (A). Cells were counted at intervals, population doublings were calculated (B), and viability (C) was determined by trypan blue dye exclusion. ( $\mathbf{D}$ and $\mathbf{E}$ ) Equal numbers of pre-B cells of each genotype were plated with (D) or without (E) IL-7. MTS assays were performed in quadruplicate at intervals (left, D and E). Total viable cell numbers (middle, $\mathbf{D}$ and $\mathbf{E}$ ) and viability (right, $\mathbf{D}$ and $\mathbf{E}$ ) were measured with trypan blue in triplicate at the indicated intervals. Data shown are representative results of 2 to 4 independent experiments from cells isolated from 2 separate litters generated by different parents. Error bars indicate the SD. For $\mathbf{E},{ }^{*} P<0.0001$, $\# P<0.0004$, and $\$ P<0.0009$, by 1 -way ANOVA.

able for lymphocyte survival due to a lack of defects in $B c l-w$-null mice (6-8). However, we directly tested whether BCL-W contributed to the survival of $B$ cells by generating cultures of primary pre-B

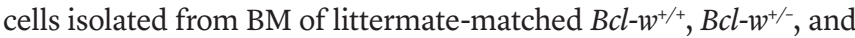
$\mathrm{Bcl}-\mathrm{w}^{-/-}$mice. BCL-W protein was readily detectable in WT pre-B cells (Figure 1A). BCL-W levels decreased by approximately half with loss of 1 allele of $B c l-w$ and were absent in the $B c l-w$-null pre-B cells. The levels of other antiapoptotic BCL-2 family members were unaltered in the $B c l-w$-deficient cells (Figure 1A). The growth rates and viability of the 3 genotypes of pre- $B$ cells cultured under normal conditions were indistinguishable from each other (Figure 1, B-D). However, loss of $B c l-w$ affected cell survival following withdrawal of IL-7, an essential cytokine. Specifically, Bcl-w-null pre-B cells had significantly reduced cell expansion following IL-7 withdrawal, due to decreased cell numbers and viability (Figure 1E). Bcl-w heterozygous pre- $\mathrm{B}$ cells showed an intermediate reduction in growth and survival compared with WT and Bcl-w ${ }^{-/}$pre-B cells after IL-7 withdrawal (Figure 1E). These results indicate that a loss of Bcl-w potentiates the negative consequences of cytokine deprivation in $\mathrm{B}$ cells and that BCL-W contributes to B cell survival.

$M Y C$-induced apoptosis is augmented by loss of $B C L-W$. To determine whether loss of $B c l-w$ would confer sensitivity to other apop- totic stimuli such as hyperproliferative signals from oncogenes like MYC, we induced the expression of a 4-hydroxytamoxifeninducible (4-OHT-inducible) form of MYC (MYCER) (14) in primary pre-B cell cultures from littermates (Figure 2A). Following activation of MYCER with 4-OHT, we observed a robust apoptotic response in pre-B cells lacking both alleles of $B c l-w$. There was a decrease in cell growth (Figure 2A), total cell numbers (Figure 2B), and viability (Figure $2 \mathrm{C}$ ) in the MYCER-activated $\mathrm{Bcl}-\mathrm{w}^{-1-}$ pre-B cells compared with WT pre-B cells. Moreover, the $B c l$-w-null pre-B cells showed an increase in annexin V-positive cells (Figure 2D) and a more rapid appearance of cleaved caspase 3 (Figure 2E) following MYCER activation. We observed a modest, but significant, decrease in cell growth and survival in $B c l-w^{+/-}$pre-B cells compared with WT pre-B cells following MYCER activation (Figure 2, A-E). These data demonstrate that BCL-W is critical for B cell survival in the presence of MYC dysregulation.

To further test the role of BCL-W in MYC-induced apoptosis in $\mathrm{B}$ cells, we derived pre- $\mathrm{B}$ cells from the $\mathrm{BM}$ of littermate-matched $B c l-w^{+/+}, B c l-w^{+/}$, and $B c l-w^{-/-} E \mu-M Y C$ mice, which overexpress MYC specifically in B cells (4), prior to any sign of lymphoma development. Within 12 days after explantation into culture, pre-B cells $\left(\mathrm{B}_{2} 2 \mathrm{O}^{+} \mathrm{CD} 19^{+} \mathrm{CD} 43^{-} \mathrm{IgM}^{-}\right)$dominated the cul- 
A

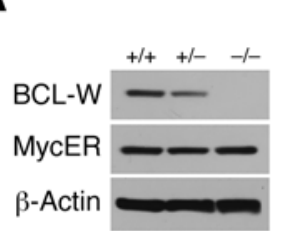

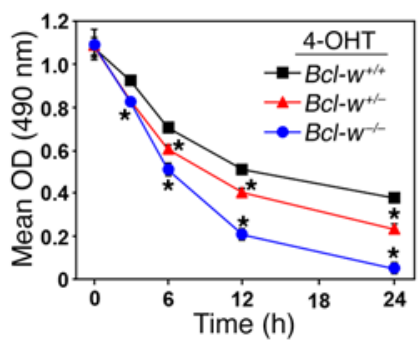

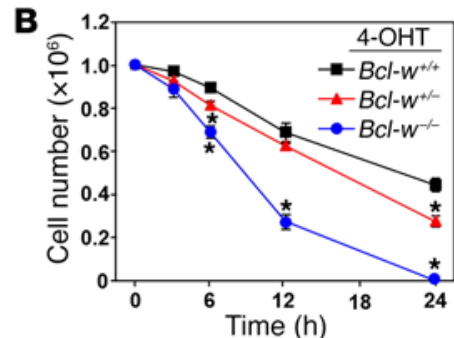

C

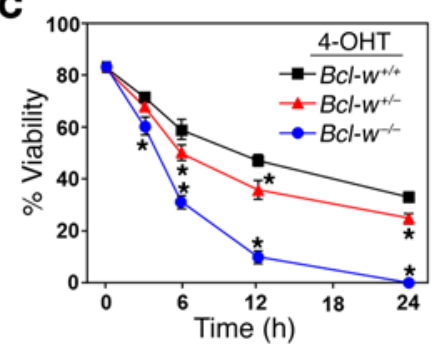

D

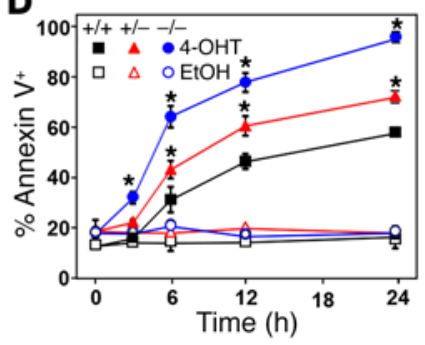

E

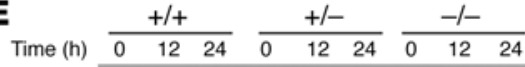

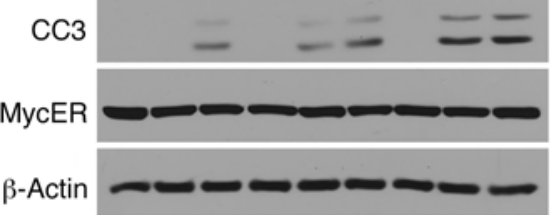

$\mathbf{F}$
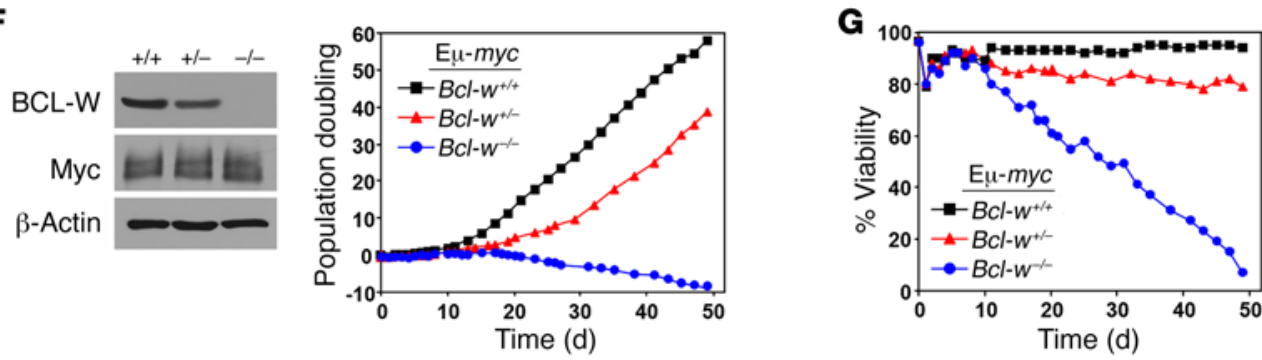

H

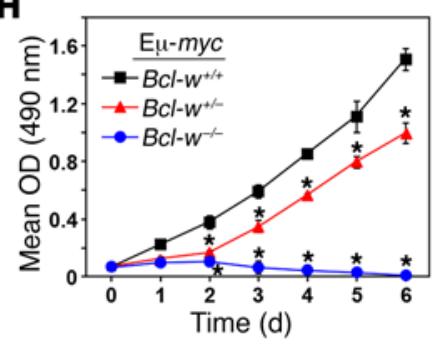

I

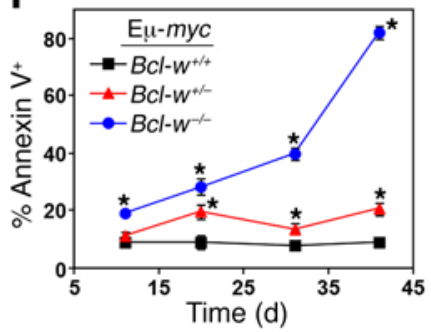

J

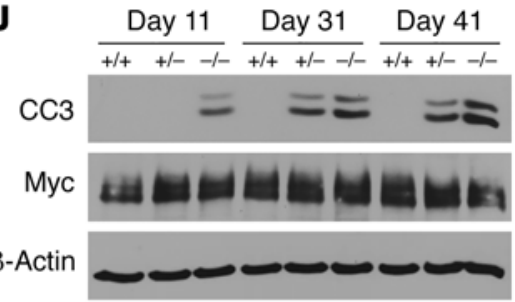

Figure 2. MYC-induced apoptosis is augmented by loss of $\mathbf{B c l - w}$. (A-E) BM-derived pre-B cells from $B c l-w^{+/+}, B c l-w^{+/-}$, and $B c l-w^{-/-}$littermates were infected with the 4-OHT-inducible MYCER. Western blot analysis was performed (A). Equal numbers of cells of each genotype were placed into culture. Following the addition of 4-OHT to activate MYCER, MTS assays (A) were performed in quadruplicate, total viable cell numbers (B) and viability (C) were determined using trypan blue dye (in triplicate), and annexin V positivity (in triplicate) (D) and caspase 3 cleavage (CC3) (E) were evaluated. (F-J) BM from Bcl-w ${ }^{+/+}$E $\mu-M Y C$, $B C l-w^{+/-} E \mu-M Y C$, and $B C l-w^{-/-} E \mu-M Y C-T g$ littermates prior to any detectable lymphoma was placed into culture on day 0 , and cells were counted at intervals. Western blot analysis was performed on the pre-B cells that grew out of the cultures (F). Population doublings were calculated (F) and viability was determined by trypan blue dye exclusion (C). (H) Equal numbers of pre-B cells of each genotype were plated and MTS assays (quadruplicate) performed. (I and J) At the indicated intervals, cells were collected and annexin $V$ positivity (I) and cleaved caspase 3 (J) assessed. Data shown are representative of 3 to 4 independent experiments using cells isolated from 3 separate litters generated by different parents. Error bars indicate the SD. ${ }^{*} P<0.0001$ for $\mathbf{A},{ }^{*} P<0.029$ for $\mathbf{B},{ }^{*} P<0.003$ for $\mathbf{C},{ }^{*} P<0.028$ for $\mathbf{D}$ (comparing 4-OHT-treated samples), ${ }^{*} P<0.0001$ for $\mathbf{H}$, and ${ }^{*} P<0.0002$ for $\mathbf{I}$, by 1 -way ANOVA.

tures for all 3 genotypes (Supplemental Figure 1; supplemental material available online with this article; https://doi.org/10.1172/ JCI89486DS1), indicating that loss of $B c l-w$ did not impair the ability of BM cells to differentiate into pre-B cells when MYC was overexpressed. However, when we evaluated pre-B cell growth by assessing the net population doublings beginning the day BM was isolated, we determined that $B c l-w^{-/} \mathrm{E} \mu-M Y C$ pre-B cells were unable to expand in culture (Figure $2 \mathrm{~F}$ ) due to decreased viability (Figure 2G). Even loss of $1 \mathrm{Bcl}$-w allele was sufficient to impair E $\mu-M Y C$ pre-B cell growth and survival (Figure 2, F and G). We obtained similar results when equal numbers of $\mathrm{E} \mu-M Y C$ pre-B cells from each genotype were cultured and evaluated each day for 6 days (Figure $2 \mathrm{H}$ ).

At intervals throughout the duration of these experiments, we assessed $\mathrm{E} \mu-M Y C$ pre-B cells of each genotype for characteristics of apoptosis. We determined that loss of $\mathrm{Bcl}$-w led to a significant increase in apoptosis over time compared with the WT counterpart, as evidenced by an increased number of annexin V-positive cells (Figure 2I) and the appearance of cleaved caspase 3 (Figure 2J). In addition, $B c l-w^{+/-} \mathrm{E} \mu-M Y C$ pre-B cells also showed an ele- 

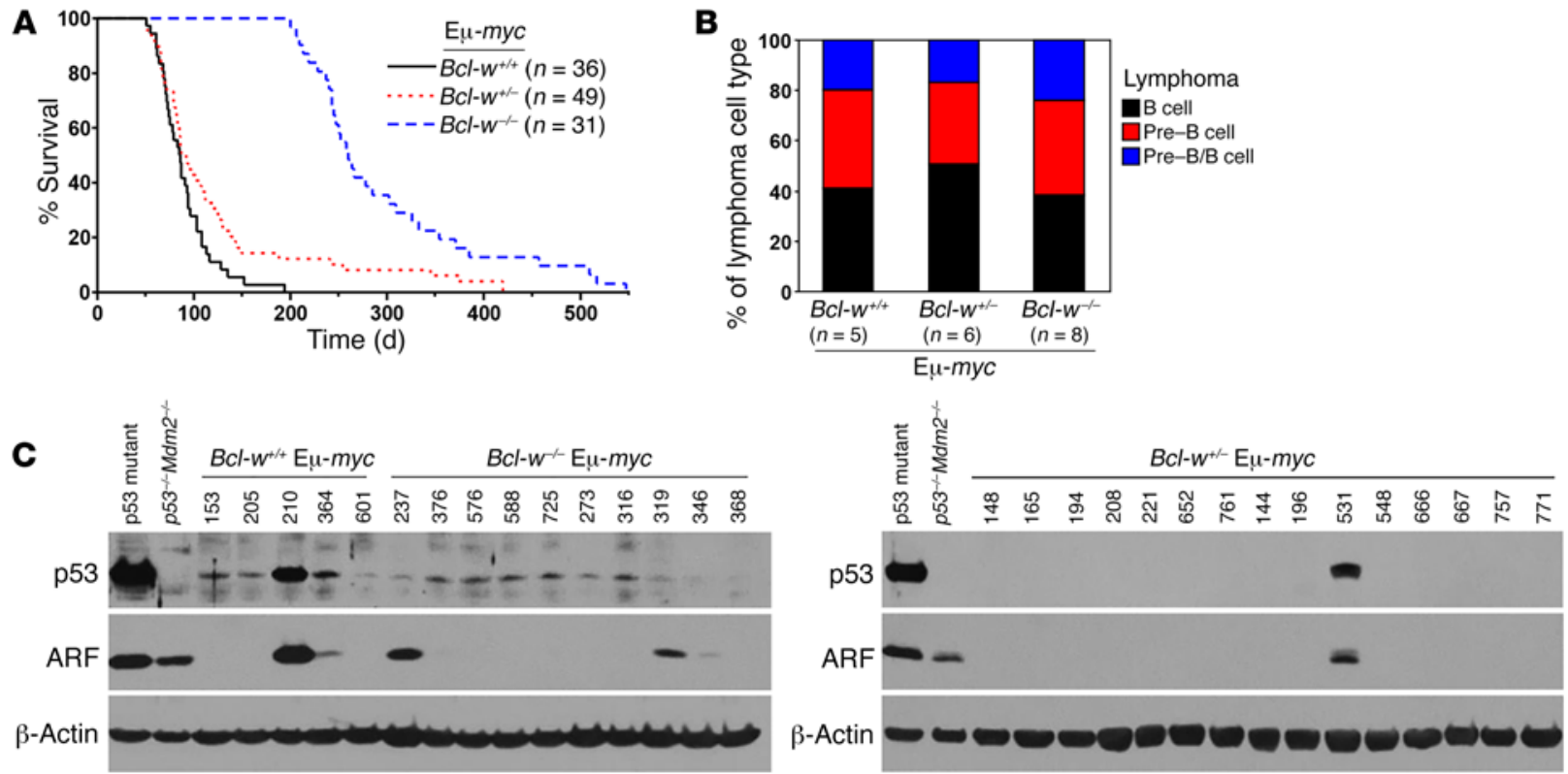

Figure 3. Loss of $\mathbf{B c l}$-w profoundly delays MYC-driven lymphomagenesis. (A) Kaplan-Meier survival curves of $B c l-w^{+/+} E \mu-M Y C, B c l-w^{+/-} E \mu-M Y C$, and $B c l-w^{-/-} E \mu-M Y C-T g$ mice. $P<0.0001$, comparing all 3 genotypes with each other; $P<0.0001$, comparing $B c l-w^{-/-} E \mu-M Y C$ with $B c l-w^{+/+} E \mu-M Y C ; P=0.0338$, comparing $\mathrm{BCl}-\mathrm{w}^{+/-} \mathrm{E} \mu-\mathrm{MYC}$ with $\mathrm{BCl}-\mathrm{w}^{+/+} \mathrm{E} \mu-\mathrm{MYC}$, by log-rank test. (B and $\mathbf{C}$ ) Lymphomas of the indicated genotypes were subjected to (B) immunophenotyping to determine the type of lymphoma that emerged and (C) Western blotting to determine p53 and ARF status. Controls included a lymphomacontaining mutant $\mathrm{p} 53$ and $p 53^{-/-}$Mdm2 $2^{-/-}$MEFs. Representative Western blots are shown; additional blots are shown in Supplemental Figure 4A. Summarized results of Western and Southern blotting are provided in Table 1.

vated apoptotic index, as these cultures had increased annexin $\mathrm{V}$ staining (Figure 2I) and caspase 3 cleavage (Figure 2J) compared with $\mathrm{Bcl}-\mathrm{w}^{+/+} \mathrm{E} \mu-\mathrm{MYC}$ pre-B cell cultures. These results further demonstrate that BCL-W has an essential role in B cell survival and inhibition of MYC-induced apoptosis.

To assess whether the effects of loss of $B c l$-w following MYC dysregulation were B cell specific, we evaluated MYCERexpressing murine embryonic fibroblasts (MEFs). First, we assessed whether a deficiency in $B c l-w$ altered the response to MYCER activation under normal growth conditions (full serum). While WT MEFs had decreased growth and viability and increased apoptosis following MYCER activation, the effects were more robust in $B c l-w$-null MEFs (Supplemental Figure 2, $\mathrm{A}-\mathrm{F})$. The apoptotic response was also accelerated in $\mathrm{Bcl}-\mathrm{w}$ heterozygous MEFs following MYCER activation compared with the response observed in $\mathrm{Bcl}-\mathrm{w}^{+/+}$MEFs (Supplemental Figure 2, A-F). Under serum-free conditions, $B c l-w$-null MEFs rapidly underwent apoptosis following MYCER activation (Supplemental Figure 2, A-C). After only 24 hours of MYCER activation, no viable cells remained in the $\mathrm{Bcl}-\mathrm{w}^{-/-}$MEF cultures, whereas a third of their WT counterparts were still viable. Together, these results show that BCL-W is not only important for the survival of B cells, but also contributes significantly to the survival of nonhematopoietic cells in response to dysregulated MYC.

Loss of BCL-W profoundly delays MYC-driven lymphoma development. Given that loss of $\mathrm{Bcl}$-w substantially increased the susceptibility of B cells to MYC-induced apoptosis, we postulated that a deficiency in $\mathrm{Bcl}$-w would negatively affect MYC-driven B cell lymphoma development. To test this hypothesis, we generated a cohort of littermate-matched $\mathrm{E} \mu-M Y C-\mathrm{Tg}$ mice with 2, 1, or no $B c l$-w alleles. $B c l-w^{-/} \mathrm{E} \mu-M Y C$ mice had an extremely long delay in lymphoma development (Figure 3A; $P<0.0001$, log-rank test). This delay in MYC-induced lymphomagenesis resulted in a mean survival of 298.5 days for $B c l-w^{-/-} \mathrm{E} \mu-M Y C$ mice, which was 3.3 times longer than the mean survival of their $B c l-w^{+/+} \mathrm{E} \mu-M Y C$ littermates (90.0 days). Of note, all of the $B c l-w^{+/+} \mathrm{E} \mu-M Y C$ mice developed lymphoma and were sacrificed before the first $B c l-w^{-/-}$ $\mathrm{E} \mu-M Y C$ mouse presented with lymphoma. Loss of just $1 \mathrm{Bcl}-\mathrm{w}$ allele also significantly delayed MYC-induced B cell lymphoma development (Figure 3A; $P=0.0338$, log-rank test; mean survival of 120.7 days). Immunophenotyping revealed no difference in the type of B cell lymphomas that arose. Specifically, all 3 genotypes developed lymphomas characteristic of those that develop in $\mathrm{E} \mu-M Y C-\mathrm{Tg}$ mice (15), with a similar distribution of pre-B cell $\left(\mathrm{B} 22 \mathrm{O}^{+} \mathrm{CD} 19^{+} \mathrm{CD} 43^{-} \mathrm{IgM}^{-}\right)$, B cell $\left(\mathrm{B} 22 \mathrm{O}^{+} \mathrm{CD} 19^{+} \mathrm{CD}^{-} 3^{-} \operatorname{IgM}^{+}\right)$, and pre-B/B cell lymphomas (Figure $3 \mathrm{~B}$ ), including lymphomas that arose early or late. Therefore, a deficiency in $B c l-w$ caused a delay in B cell lymphoma development, but did not alter the type of lymphoma that emerged.

\section{Table 1. Reduced ARF and p53 inactivation in Bcl-w-deficient Iymphomas}

$\begin{array}{lccc}\text { Genotype } & p 53 \text {-mutated } & \text { p53-deleted } & \text { Arf-deleted }^{\mathrm{B}} \\ B C l-\mathrm{w}^{+/+} \mathrm{E} \mu-M Y C & 2 \text { of } 10(20 \%) & 0 \text { of } 10(0 \%) & 2 \text { of } 10(20 \%) \\ B C l-\mathrm{w}^{+/-} \mathrm{E} \mu-M Y C & 1 \text { of } 15(7 \%) & 0 \text { of } 15(0 \%) & 2 \text { of } 15(13 \%) \\ B C l-\mathrm{w}^{-/-} \mathrm{E} \mu-M Y C & 1 \text { of } 20(5 \%) & 0 \text { of } 20(0 \%) & 2 \text { of } 20(10 \%)\end{array}$

${ }^{A}$ Determined by sequencing; ${ }^{B}$ determined by Southern blotting 

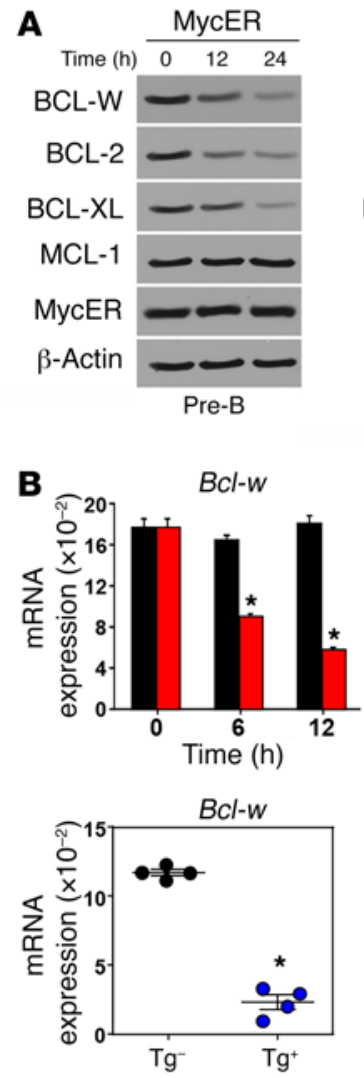

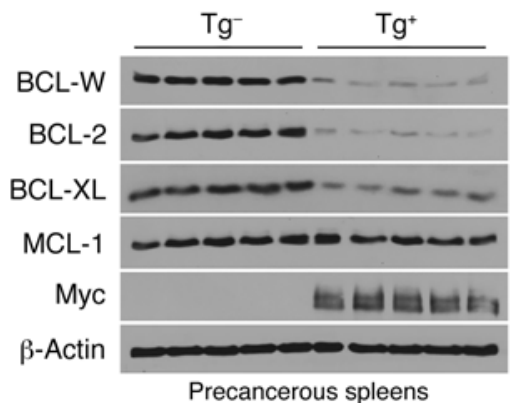

Precancerous spleens
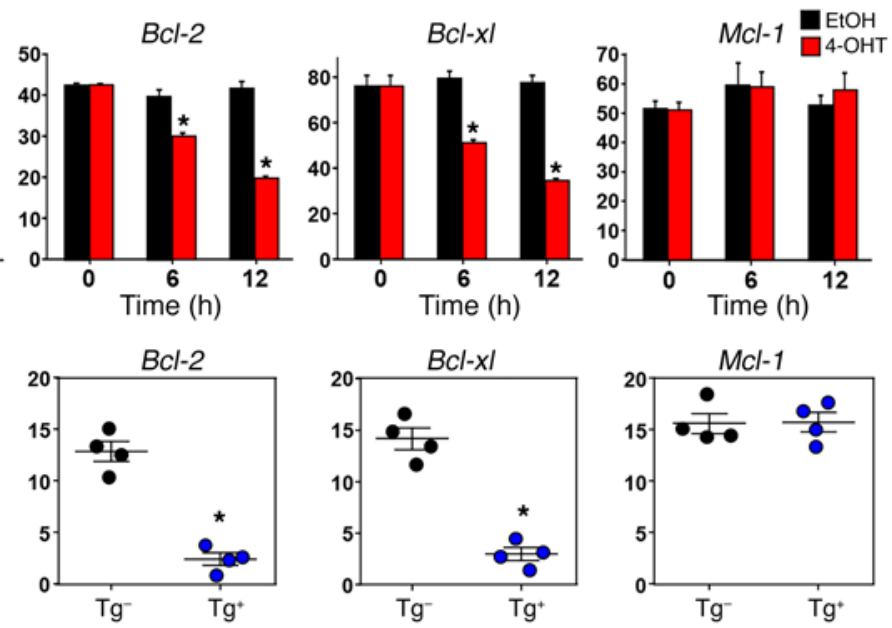

C

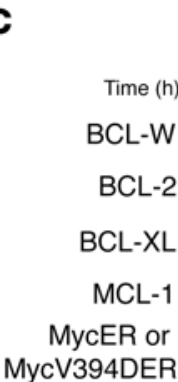

$\beta$-Actin
D

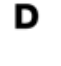

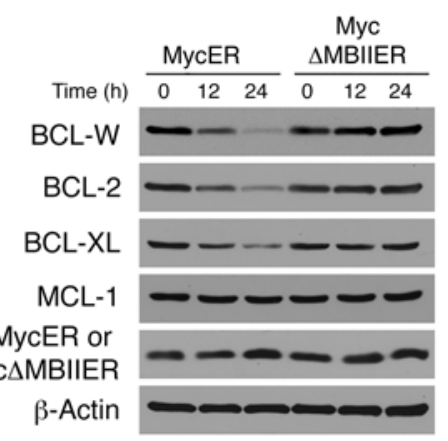
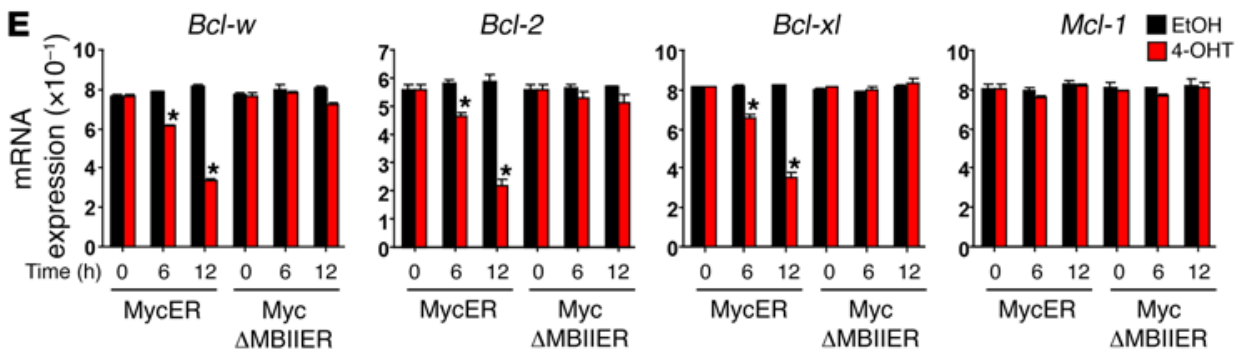

Figure 4. MYC suppresses BCL-W expression in normal cells. At intervals following the addition of 4-OHT, WT pre-B cells (A, left) and $p 53-$ null MEFs (C-E) expressing either WT MYCER (A, left, B, top, C, and D), MYCV394D-ER (C), or MYC $\triangle$ MBII-ER ( $\boldsymbol{D}$ and $\mathbf{E}$ ) were Western blotted for the indicated proteins (A, C, and $\mathbf{D})$, and mRNA levels were measured in triplicate by qRT-PCR (B, top, and E). Precancerous splenocytes from $\mathrm{E} \mu-M Y C$ $\mathrm{Tg}\left(\mathrm{Tg}^{+}\right)$and non-Tg littermates $\left(\mathrm{Tg}^{-}\right)$were subjected to Western blotting (A, right) and qRT-PCR (in triplicate, B, bottom; $n=4$ of each). mRNA expression was normalized to $\beta$-actin and presented as $2^{-\Delta \Delta \mathrm{Ct}}$. Data shown are representative of 2 to 4 independent experiments. Error bars indicate the SEM. ${ }^{*} P<0.0071$ (B) and ${ }^{*} P<0.005(\mathbf{E})$, by $t$ test.

numbers at all stages of development in the BM of $B c l$-w-deficient $\mathrm{E} \mu-M Y C$ mice (Supplemental Figure 3B). Evaluation of littermate-matched, non$\mathrm{Tg}$ mice showed analogous results among the 3 genotypes (Supplemental Figure 3, A and B). Therefore, a deficiency in $B c l$-w does not appear to alter $B$ cell development in mice with normal or increased levels of MYC.

MYC overexpression selects for inactivation of the ARF/p53 tumor-suppressor pathway during lymphomagenesis, and alterations in proteins that influence apoptosis can change the frequency of ARF and/or p53 inactivation (16-18). To assess whether loss of $B c l-w$ affected the frequency of ARF and/or p53 inactivation, we performed Western (Figure $3 \mathrm{C}$ and Supplemental Figure 4A) and Southern (Supplemental Figure 4B) blot analyses of lymphomas from mice of all 3 genotypes. As mutations in p53 typically result in protein overexpression (16), we performed sequencing of p53 on samples that showed increased levels of $\mathrm{p} 53$ protein. We also sequenced p53 in lym-

To determine whether the protracted latency of lymphoma development in mice lacking $B c l-w$ was due to a deficiency in B cells, we performed immunophenotyping on 4 independent sets of littermate-matched precancerous $\mathrm{E} \mu-M Y C$ mice to evaluate B cell populations. The data show no difference in the number of $B$ cells in the spleens of $B c l-w^{-/} \mathrm{E} \mu-M Y C$ or $B c l-w^{+/-} \mathrm{E} \mu-M Y C$ mice compared with their WT E $\mu-M Y C$ littermates (Supplemental Figure 3A). Similarly, there was no discernible difference in B cell phomas with increased ARF protein due to the negative feedback regulation of ARF by p53. Only lymphomas with elevated $\mathrm{p} 53$ protein harbored mutations in the DNA-binding domain of $\mathrm{p} 53$, the most frequently mutated region in p53 (19). Loss of Bcl-w resulted in less frequent mutations of $\mathrm{p} 53$ (Table 1). $p 53$ deletions are rare in $\mathrm{E} \mu-M Y C-\mathrm{Tg}$ mice, and we detected no $p 53$ deletions in any of the lymphomas from mice of the 3 genotypes (Table 1 and Supplemental Figure 4B). We observed a decrease in the frequency of 

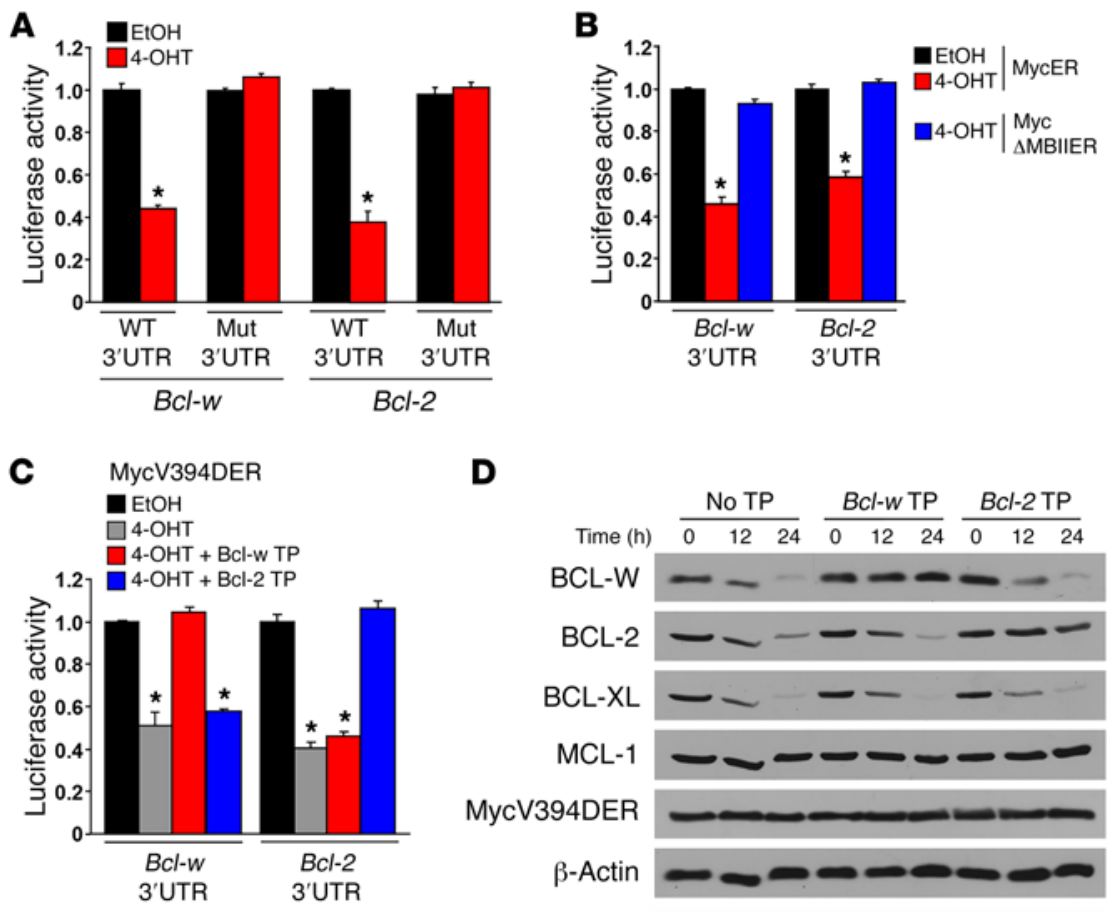

Figure 5. BCL-W expression is regulated by MYC through transcriptional upregulation of the miR-15 family. (A-C) A luciferase reporter containing the $3^{\prime}$-UTR of $B c l-w$ with a WT or mutated (Mut) miR-15 family binding site was transfected into $p 53^{-/-} \mathrm{MEFs}$ expressing MYCER (A), MYC $\triangle M B I I-E R(B)$, or MYCV394D-ER (C). Luciferase activity was measured in triplicate 48 hours after the addition of vehicle control (EtOH) or 4-OHT. A luciferase reporter containing either the WT or mutated miR-15 family binding site of the $3^{\prime}$-UTR of $\mathrm{BCl}-2$ served as a positive control for A-C. A $\beta$-gal reporter was cotransfected for normalization purposes. (C and $\mathbf{D})$ miR-15 family binding sites in the $3^{\prime}$-UTR of $B c l-W$ and $B c l-2$ were blocked with site-specific small molecules (Target Protectors [TP]) 24 hours prior to MYCV394D-ER activation. Following the addition of 4-OHT, luciferase activity (C) and protein levels (D) were assessed in the presence or absence of the $\mathrm{BCl}-\mathrm{w}$ and $\mathrm{BCl}-2$ Target Protectors. Data shown are representative of 2 to 4 independent experiments. Error bars indicate the $\mathrm{SD}$. ${ }^{*} P=0.0017$ (A), by $t$ test; ${ }^{*} P<0.0001$ (B and C), by 1-way ANOVA.
Arf deletions in lymphomas that lacked $B c l-w$ (Table 1 and Supplemental Figure 4B). Together, these results suggest that, without $B c l-w$, the selective pressure to inactivate the ARF/p53 pathway was reduced during lymphomagenesis.

MYC suppresses BCL-W expression. MYC activates apoptosis by activating $\mathrm{p} 53$, but also by suppressing the expression of BCL-2 and/or BCL-XL independently of p53 (20-23). We investigated whether BCL-W expression was regulated by MYC by expressing MYCER in pre-B cells. Activation of MYCER with the addition of 4-OHT decreased BCL-W as well as BCL-2 and BCL-XL protein levels (Figure 4A, left). Consistent with previous reports $(20,23)$, protein levels of the antiapoptotic BCL-2 family member MCL-1 did not change following MYCER activation (Figure 4A, left). As a separate, independent test of MYC regulation and to determine whether MYC regulates BCL-W expression in vivo, we evaluated spleens from precancerous $\mathrm{E} \mu-M Y C$ mice and littermate-matched non-Tg spleens. E $\mu-M Y C$ spleens had reduced BCL-W protein levels as well as decreased BCL-2 and BCL-XL, but MCL-1 levels were unchanged compared with levels detected in the littermate-matched non-Tg spleens (Figure 4A, right). Evaluation of $B c l-w$ mRNA by quantitative real-time PCR (qRT-PCR) showed that, following MYCER activation in pre-B cells, $B c l$-w mRNA levels decreased (Figure 4B, top panels). In addition, spleens from precancerous $\mathrm{E} \mu-M Y C$ mice had reduced levels of $B c l-w$ mRNA compared with spleens from non-Tg littermates (Figure $4 \mathrm{~B}$, bottom panels). Taken together, MYC induces a decrease in BCL-W mRNA and protein levels in B cells.

To determine whether the MYC-induced decrease in BCL-W was cell-type specific, we also assessed WT MEFs expressing MYCER. Analogous to the results we obtained in pre-B cells, activation of MYCER in these nonhematopoietic cells decreased BCL-W levels (Supplemental Figure 5). Likewise, BCL-2 and BCLXL levels were also reduced following MYCER activation (Sup- plemental Figure 5). Therefore, the MYC-induced decrease in BCL-W also occurred in primary nonhematopoietic cells, indicating that this was a generalizable consequence of MYC activation.

Suppression of BCL-W expression is independent of MIZ-1 and p53, but requires MYC transcriptional activity. To gain insight into the mechanism by which MYC was modulating BCL-W expression, we performed a series of experiments using mutants of MYC. MYC can suppress the expression of some genes by inhibiting the transcriptional activator MIZ-1 (24). Using a mutant of MYCER containing a point mutation interrupting the MYC:MIZ-1 interaction (MYCV394D-ER) (25), we determined that BCL-W protein levels decreased in cells expressing MYCV394D-ER to an extent similar to that seen in cells with WT MYCER following activation with 4-OHT (Figure 4C). We also observed analogous reductions of BCL-2 and BCL-XL, but not of MCL-1 (Figure 4C). To evaluate whether a transcriptionally competent MYC was required to suppress BCL-W protein expression, we used a mutant of MYCER lacking the MYC box II domain, which is crucial for MYC transcriptional activity (MYCAMBII-ER) (24). Upon addition of 4-OHT, we observed a decrease in BCL-W protein only in cells expressing the WT MYCER, but not in cells expressing the transcriptionally impaired MYC $\triangle$ MBII-ER mutant (Figure 4D). The decrease in BCL-W protein expression was reflected at the mRNA level, as a reduction in $\mathrm{Bcl}$-w mRNA only occurred in cells expressing WT MYCER, but not the transcriptionally impaired MYC $\triangle$ MBII-ER (Figure 4E). These data demonstrate that MYC transcriptional activity is required to downregulate BCL-W expression. Furthermore, the experiments using MYCER mutants were performed in cells lacking $p 53$ (Figure 4, C-E), indicating that the MYC-induced decrease in BCL-W expression occurs independently of $\mathrm{p} 53$.

$B C L-W$ expression is modulated by the MYC-regulated miR-15 family. Upon evaluation of publically available ENCODE MYC ChIP-sequencing (ChIP-seq) data (26), MYC was not present at 
A

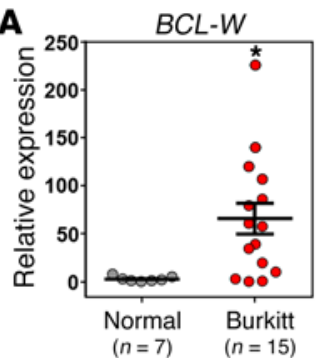

C

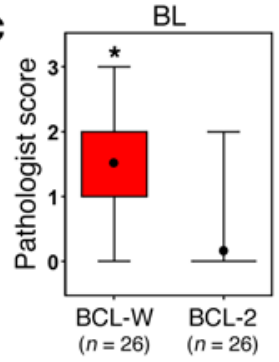

$B C L-2$
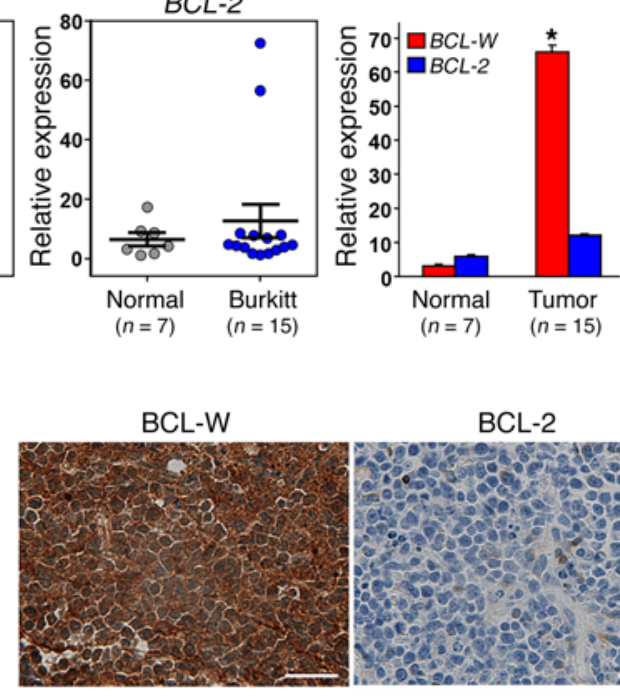

B

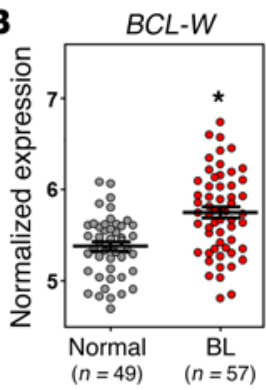

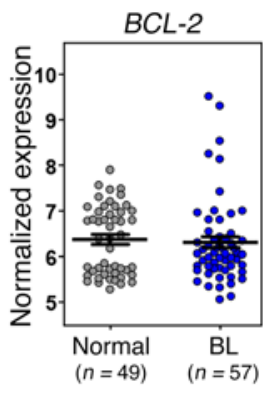

D

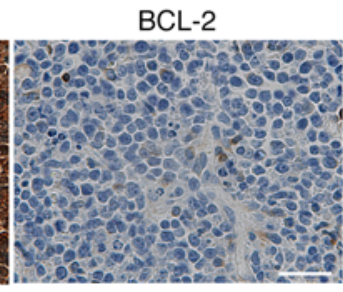

DLBCLs

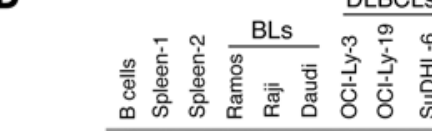

BCL-W

$\beta$-Actin

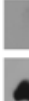

Figure 6. BCL-W expression is selected for in human BL. (A) qRT-PCR analysis (in triplicate) of samples from patients with BL ( $n=15)$ compared with normal lymphoid control tissue $(n=7)$. mRNA levels were normalized to $\beta$-actin and presented as $2^{-\Delta \Delta C t}$. Bars represent the mean $\pm S E M$. ${ }^{*} P=9.1 \times 10^{-4}$, by $t$ test. (B) Microarray gene expression-profiling data for BCL-W and BCL-2 mRNA in BL $(n=57)$ compared with normal B cells ( $n=49)$. Bars represent the mean \pm SEM. Each circle represents 1 sample, and the $y$ axis represents normalized expression of $B C L-W$ and $B C L-2$. ${ }^{*} P=1.8 \times 10^{-8}$, by $t$ test. The data sets analyzed are listed in Supplemental Table 1. (C) Immunohistochemical analysis for BCL-W and BCL-2 protein was performed on samples from patients with BL $(n=26)$. Box and whisker plots of pathologist scores and representative images of BCL-W and BCL-2 staining from the same tumor sample are shown. Original magnification, $\times 40$; scale bars: $200 \mu \mathrm{m}$. ${ }^{*} P<0.0001$, by $t$ test. For the box and whisker plots, the box represents the $25^{\text {th }}$ and $75^{\text {th }}$ percentiles, the line indicates the median, the circle indicates the mean, and the whiskers represent the maximum and minimum. (D) Protein expression was assessed by Western blotting for the indicated proteins in BL and DLBCL cell lines. Controls included tissue from 2 human spleens and purified B cells from human peripheral blood.

the $\mathrm{Bcl}$-w promoter region in hematopoietic or nonhematopoietic cell lines (Supplemental Figure 6). Therefore, we postulated that MYC may be indirectly altering $B c l$-w expression through miR. As we (Supplemental Figure 7) and others (27-29) have shown, Bcl-w mRNA is targeted and negatively regulated by the miR-15 family. Recently, we reported that MYC transcriptionally upregulates this miR family as a mechanism to downregulate $\mathrm{Bcl}-2$ expression (22, 23). To test whether MYC activation leads to miR-15 family targeting of $B c l-w$, we used a luciferase-based assay to detect direct binding of the miR-15 family to its predicted target site in the $3^{\prime}$-UTR of $B c l-w$ following MYC-induced transcriptional upregulation. Specifically, luciferase reporters harboring either a WT miR-15 family binding site of the $B c l-w$ 3'-UTR or a mutated version were introduced into $p 53^{-/-}$MEFs expressing the 4-OHT-inducible form of MYCER. Upon activation of MYCER, we detected a decrease in luciferase activity in cells containing the WT miR-15 family binding site, but not the mutated miR-15 family binding site (Figure 5A). As a positive control, we evaluated cells with WT or mutated miR-15 family binding sites in the 3'-UTR of Bcl-2 and obtained results analogous to those with the $B c l-w$ 3'-UTR (Figure 5A). These results indicate that MYC induces the expression of miR15 family members, as we have recently reported (23), which then directly bind and negatively regulate $\mathrm{Bcl}$-w expression.

To further assess the mechanism by which MYC regulates the miR-15 family to target $B c l-w$, we used the transcriptionally incompetent form of MYC. p53-null MEFs expressing either the WT MYCER or the deletion mutant MYCAMBII-ER were transfected with a luciferase reporter containing the WT miR-15 family binding site of either the Bcl-w 3'-UTR or, as a positive control, the Bcl-23'-UTR. Following activation of WT MYCER, luciferase activity decreased in cells containing the $\mathrm{Bcl}$ - $\mathrm{w}$ or $\mathrm{Bcl}-23^{\prime}$-UTR (Figure 5B). In contrast, after addition of 4-OHT to the MYCAMBII-ER-expressing cells, we detected no change in luciferase activity for either luciferase reporter (Figure 5B), indicating that a transcriptionally competent MYC is required for the miR-15 family-mediated decrease in $\mathrm{Bcl}$-w expression.

We further validated this MYC-regulated miR-mediated mechanism by transfecting p53-null MEFs expressing the MYCER mutant unable to interact with MIZ-1 (MYCV394D-ER) with modified RNA molecules (Target Protectors) designed to block endogenous miR-15 family members from binding their target site in the $3^{\prime}$-UTR of $B c l-w$. In addition to the Target Protectors, the MYCV394D-ER-expressing cells received luciferase reporters containing the miR-15 family binding site for either the 3'-UTR of $B c l-w$ or, as a control, $B c l-2$. In the absence of any Target Protectors, MYCV394D-ER activation, which upregulates the miR-15 family (23), resulted in decreased luciferase activity in cells with either the $\mathrm{Bcl}$-w or $\mathrm{Bcl}-2$ luciferase reporters (Figure $5 \mathrm{C})$. However, when the miR-15 family binding site of the $B c l-w$ or Bcl-2 3'-UTR was blocked by their respective Target Protectors, luciferase activity remained unchanged (Figure $5 \mathrm{C}$ ). As an additional control to test the specificity of our system, cells containing the $B c l-w$ or $B c l$-2 3'-UTR luciferase reporters were transfected with the opposite Target Protector. Following MYCV394D-ER activation with 
Table 2. Characteristics of BL and DLBCL patients for IHC

\begin{tabular}{lcc} 
Characteristic & BL & DLBCL \\
$\begin{array}{l}\text { Patients } \\
\text { Median age, yr (range) }\end{array}$ & $n=26$ & $n=57$ \\
$\begin{array}{l}\text { Sex, } n \text { (percentage) } \\
\text { Male }\end{array}$ & $94(10-20)$ & \\
\hline Female & $17(65.4 \%)$ & $35(61.4 \%)$ \\
\hline NA & $8(30.8 \%)$ & $19(33.3 \%)$ \\
BCL-W, $n$ (percentage) & $1(3.8 \%)$ & $2(3.5 \%)$ \\
\hline 0 & & \\
\hline $1^{A}$ & $3(11.5 \%)$ & $7(12.3 \%)$ \\
$2^{A}$ & $11(42.3 \%)$ & $16(28.1 \%)$ \\
\hline $3^{A}$ & $7(26.9 \%)$ & $18(31.6 \%)$ \\
BCL-2, $n$ (percentage) & $5(19.2 \%)$ & $16(28.1 \%)$ \\
\hline 0 & & $15(26.3 \%)$ \\
$1^{A}$ & $22(84.6 \%)$ & $6(10.5 \%)$ \\
$2^{A}$ & $3(11.5 \%)$ & $14(24.6 \%)$ \\
\hline $3^{A}$ & $1(3.9 \%)$ & $22(38.6 \%)$ \\
\hline
\end{tabular}

${ }^{A}$ Where duplicate or triplicate samples were available, scores were averaged and rounded to the highest integer. NA, not available.

4-OHT, luciferase activity decreased to an extent that was similar to that seen with no Target Protector present (Figure 5C). In addition, MYCV394D-ER activation decreased BCL-W and BCL-2 protein expression levels in the absence of any Target Protector (Figure 5D). However, BCL-W and BCL-2 levels remained unchanged when their respective Target Protectors were present to block the miR-15 family binding sites (Figure 5D). Together, these results provide direct mechanistic data showing that MYC transcriptionally upregulates the miR-15 family members that then target and decrease the expression of $B c l-w$ and that this occurs independently of both MIZ-1 and p53.

$B C L-W$ is overexpressed in human $B L$. We recently reported that the transformation status of the cell dictates whether MYC functions as a transcriptional activator or repressor of the miR15 family (23). In normal, nontransformed cells, MYC transcriptionally upregulates the miR-15 family, which downregulates $B c l-2$ and, as we show here, $B c l$ - $w$ expression, leading to increased apoptosis. However, this apoptotic mechanism is inactivated in cancer cells, as MYC is converted from a transcriptional activator to a transcriptional repressor of the miR-15 family, which would allow $\mathrm{Bcl}-2$ and, presumably, $\mathrm{Bcl}$-w levels to be increased in cancer cells that overexpressed MYC (23).

We and others have reported that MYC-overexpressing murine lymphoma cells have reduced expression of the miR-15 family due to MYC-mediated repression $(22,23,30)$. We evaluated miR-15a expression in formalin-fixed, paraffin-embedded BL patient samples, which constitutively overexpress MYC due to a MYC translocation, a defining feature of this lymphoma (31). As expected, miR-15a levels were significantly decreased in BL patient samples compared with levels in normal controls (Supplemental Figure 8A), whereas levels of miR-17-5p, which is induced by MYC in cancer cells, were increased in the BL samples (Supplemental Figure $8 \mathrm{~B})$. Therefore, as in murine B cell lymphoma, miR-15a levels are reduced in human $\mathrm{B}$ cell lymphoma.
We next performed qRT-PCR to evaluate mRNA levels of $B C L-W$ and $B C L-2$ from RNA isolated from formalin-fixed, paraffin-embedded BL patient samples and normal lymph node controls. Strikingly, $B C L-W$ mRNA was overexpressed in the $\mathrm{BL}$ patient samples compared with expression levels detected in the normal tissue (Figure 6A and Supplemental Figure 9). Because low or undetectable BCL-2 levels are typical in BL and considered during diagnosis, $B C L-2$ levels were evaluated and showed no overexpression compared with normal tissue (Figure 6A and Supplemental Figure 9). As a separate, independent analysis of $B C L-W$ mRNA expression in human BL, we evaluated publically available microarray gene expression-profiling data (http://www.ncbi.nlm. nih.gov/geo/). Consistent with our qRT-PCR data, $B C L-W$ was significantly overexpressed in BL samples compared with either a mixed population of normal B cells or only normal germinal center $\mathrm{B}$ cells (Figure 6B and Supplemental Figure 10). We also assessed $B C L-2$ mRNA levels and determined that they were not elevated in BL cells compared with levels in normal B cells (Figure 6B). These data indicate that increased $B C L-W$ mRNA expression is selected for in human $\mathrm{BL}$, whereas $B C L-2$ is not.

To determine whether the increase in $B C L-W$ mRNA resulted in increased BCL-W protein expression, we performed immunohistochemical analyses of samples from patients with BL. Blinded scoring by a hematopathologist revealed that expression of BCL-W protein was a very frequent event in BL. Specifically, $88.5 \%$ (23 of 26) of the samples had positive staining ( $\geq 1$ pathologist score) for BCL-W (Figure 6C, Table 2, Supplemental Figure 11). Only 15.4\% (4 of 26) of the samples showed BCL-2 positivity, which was predominantly low. Additionally, BCL-W protein was overexpressed in BL cell lines compared with normal human splenic tissue and isolated B cells (Figure 6D). Evaluation of BCL-W levels in B cell lymphomas from E $\mu-M Y C-T g$ mice showed a similarly high frequency of increased BCL-W protein expression (Supplemental Figure 12). These results indicate that, in addition to murine lymphoma, BCL-W may be a significant contributor to the pathogenesis of human BL.

Targeting BCL-W in MYC-driven BL induces apoptosis. Given that $\mathrm{BCL}-\mathrm{W}$ expression is highly selected for in $\mathrm{BL}$, we tested whether targeting $B C L-W$ would affect BL cell survival. Because we have established a link between $B c l-w$ and the miR- 15 family, which is repressed in BL, we hypothesized that increasing levels of the miR15 family in these lymphoma cells would decrease BCL-W expression, leading to apoptosis. To test this, we ectopically expressed the miR-15a/16-1 cistron or empty vector as a control in 2 human BL cell lines. In both BL cell lines, ectopic expression of miR-15a/16-1 decreased BCL-W protein expression (Supplemental Figure 13A). Reduced levels of BCL-2 protein, which we previously reported occurred with ectopic expression of miR-15a/16- 1 in murine lymphoma cells (23), were also detected and served as a positive control (Supplemental Figure 13A). The forced expression of miR15a/16-1 reduced cell growth, total cell numbers, and viability (Supplemental Figure 13, B-D). Furthermore, ectopic miR-15a/16-1 expression induced apoptosis, as evidenced by the appearance of cleaved caspase 3 (Supplemental Figure 13A). Therefore, increasing expression of the miR-15 family members that target and downregulate $B C L-W$ and $B C L-2$ induces apoptosis of $B L$ cells.

As an independent approach to specifically test the dependency of BL cells on BCL-W expression, we used a doxycycline- 
A
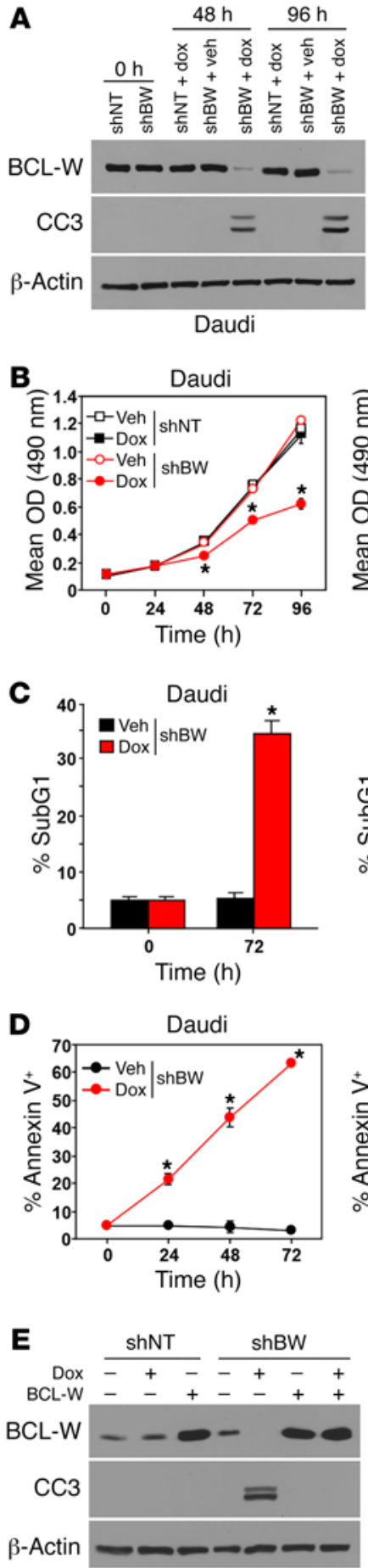
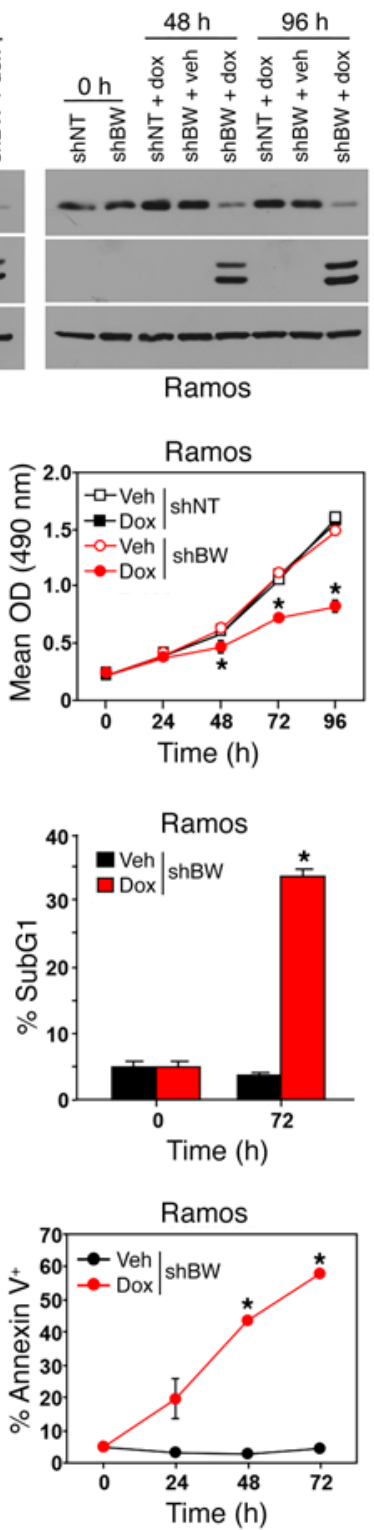

$\mathbf{F}$

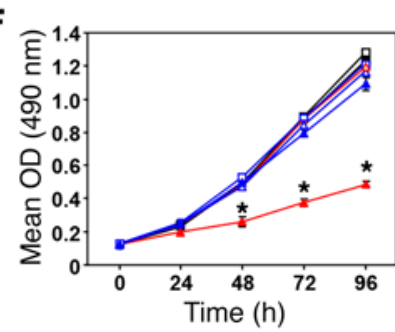

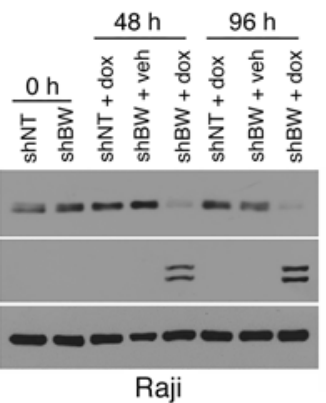
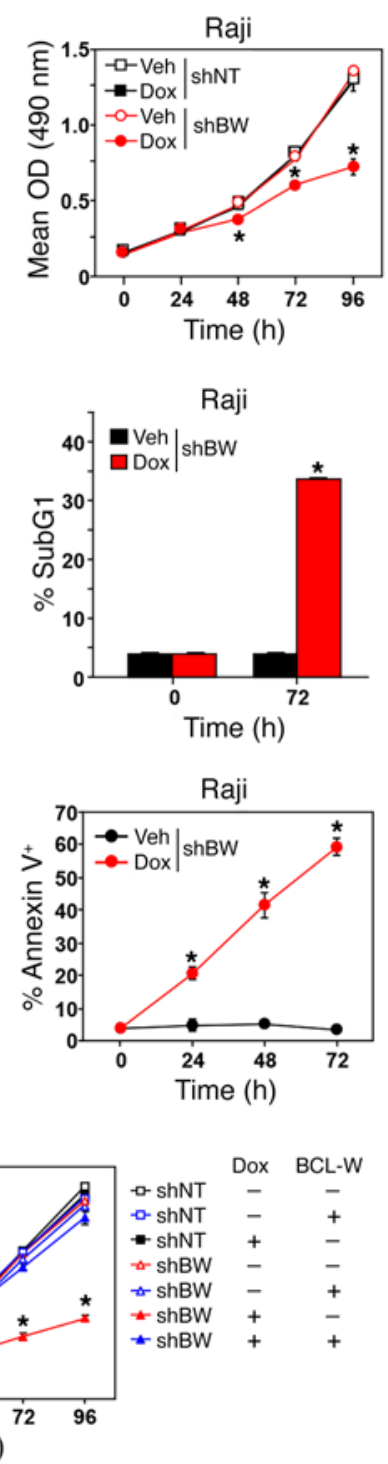

Figure 7. Targeting BCL-W induces apoptosis in human BL. (A-D) The indicated human BL cell lines were infected with a Dox-inducible lentivirus encoding either a $B C L-W$ shRNA (shBW) or nontargeting (shNT) control shRNA. Following the addition of Dox or DMSO vehicle (Veh) control, Western blotting for cleaved caspase 3 (A), MTS assays (B, in quadruplicate), and staining for subG1 DNA content (C, in triplicate) and annexin $V$ positivity (D, in triplicate) were performed. (E and F) Daudi cells were doubly infected with the Dox-inducible lentivirus expressing either a $B C L-W$ shRNA (shBW) or nontargeting (shNT) control shRNA and a retrovirus expressing $B C L-W(+)$ or empty vector control (-). Following the addition of Dox $(+)$ or DMSO vehicle control (-), Western blotting for the indicated proteins (E) and MTS assays (F, in quadruplicate) were performed. Data shown are representative of 2 to 3 independent experiments. Error bars for $\mathbf{B}-\mathbf{D}$ and $\mathbf{F}$ indicate the $\mathrm{SD}$. ${ }^{*} P<6.4 \times 10^{-4}$ (B), ${ }^{*} P<0.025$ (C), ${ }^{*} P<0.034$ (D), and ${ }^{*} P<0.0036(\mathbf{F})$, by $t$ test comparing shBW and Dox with shBW and vehicle.

sion of BCL-W did not show a decrease in growth or an increase in caspase 3 cleavage after the addition of Dox, which was only evident in BL cells expressing the $B C L-W$ shRNA alone following the addition of Dox (Figure 7, E and F). These data indicate that targeting BCL-W in BL, which selects for increased BCL-W expression, may be an effective therapeutic approach to treat BL.

As an additional test of the requirements of BCL-W for BL survival, we took a pharmacological approach. The $\mathrm{BH} 3$ mimetic compounds ABT-737 and its ana$\log$ ABT-263 directly activate apoptosis by binding and inhibiting the antiapoptotic BCL-2 proteins BCL-2, BCL-XL, and BCL-W (5). To evaluate the effect of ABT737 and ABT-263 on BL, we first determined the $\mathrm{IC}_{50}$ of each in 2 human BL cell lines (Figure 8A). The $\mathrm{IC}_{50}$ for ABT-737 and ABT-263 was $500 \mathrm{nM}$ and $1 \mu \mathrm{M}$, respectively, for Daudi cells and $250 \mathrm{nM}$ and 2.5 $\mu \mathrm{M}$, respectively, for Ramos cells. To determine whether BCL-W expression alone inducible (Dox-inducible) $B C L-W$ shRNA in 3 human BL cell lines. Following the addition of Dox to activate expression of the $B C L-W$ shRNA, BCL-W protein expression decreased (Figure 7A). This resulted in reduced cell growth in all $3 \mathrm{BL}$ cell lines (Figure 7B). The reduction in cell growth was attributed to increased apoptosis, as indicated by the increased subG1 DNA content (Figure 7C), annexin $\mathrm{V}$ positivity (Figure 7D), and caspase 3 cleavage (Figure 7A). To test whether the negative consequences of $B C L-W$ shRNA were specific and not due to off-target effects, BCL-W was ectopically expressed. BL cells with $B C L-W$ shRNA and ectopic expres- could protect the BL cells from ABT-737 and ABT-263, BCL-W was ectopically expressed (Figure 8B and Supplemental Figure 14A) and treated the $\mathrm{BL}$ cells with the $\mathrm{IC}_{50}$ of each. After 48 hours of treatment with the $\mathrm{BH} 3$ mimetics, the growth of $\mathrm{BL}$ cells expressing the control vector was significantly diminished (Figure $8 \mathrm{C}$ and Supplemental Figure 14B) as a result of increased apoptosis, as indicated by the appearance of cleaved caspase 3 (Figure $8 \mathrm{~B}$ and Supplemental Figure 14A). However, cells overexpressing BCL-W were not sensitive to ABT-737 or ABT-263, as growth of these cells remained unaffected, and no caspase 3 cleavage was detected (Fig- 
A
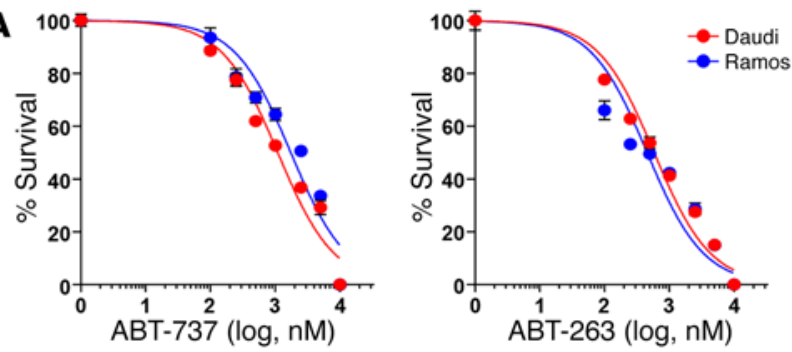

B

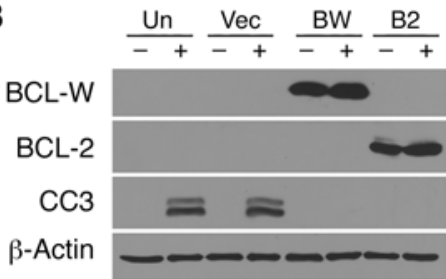

ABT-737
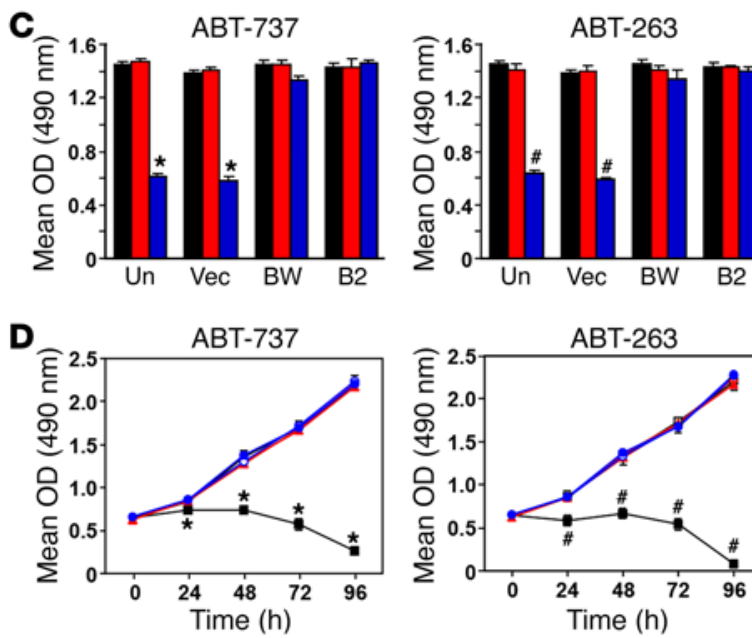

$\mathbf{E}$
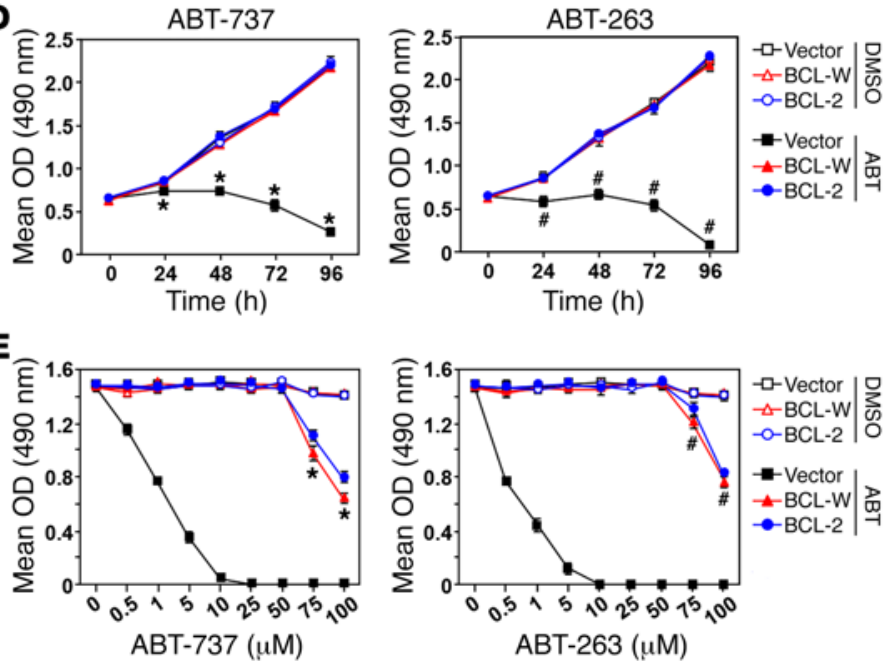

ure 8, B and C and Supplemental Figure 14, A and B). Additionally, 4-day kinetics experiments also showed that BL cells with forced overexpression of BCL-W were protected from the apoptosisinducing properties of the $\mathrm{BH} 3$ mimetics (Figure 8D and Supplemental Figure 14C). Finally, increasing concentrations of ABT-737 and ABT-263 were administered to BL cell lines to determine the maximal dose at which the cells overexpressing BCL-W would no longer be protected. BL cells with the vector control quickly succumbed to $\mathrm{BH} 3$ mimetic treatment, with all cells dying following administration of $10 \mu \mathrm{M}$ of either compound within 48 hours (Figure $8 \mathrm{E}$ and Supplemental Figure 14D). However, high concentrations $(>50 \mu \mathrm{M})$ of either compound were necessary to kill the $\mathrm{BL}$ cells overexpressing BCL-W (Figure 8E and Supplemental Figure 14D). Results similar to those with BCL-W overexpression were
Figure 8. Increased expression of BCL-W confers resistance to BL cells to BH3 mimetics. (A) The $\mathrm{IC}_{50}$ of the BH3 mimetics ABT-737 and ABT-263 for 2 human BL cell lines (Daudi and Ramos) was determined using MTS assays (in quadruplicate, 48 h). (B-E) Daudi cells remained uninfected (Un) or were retrovirally infected to express empty vector $(\mathrm{Vec}), \mathrm{BCL}-\mathrm{W}(\mathrm{BW})$, or $\mathrm{BCL}-2$ (B2). Following treatment with $\mathrm{ABT}-737$ $(+)$, ABT-263 (+), or DMSO vehicle control (-) at the determined IC ${ }_{50}$ (B-D) or the indicated concentrations (E) for 48 hours (B, C, and E) or the indicated intervals (D), Western blotting for cleaved caspase 3 (B) and MTS assays (C-E, in quadruplicate) were performed. Data shown are representative of 2 independent experiments for both inhibitors. For data in $\mathbf{D}$ and $\mathbf{E}$, experiments with inhibitors were performed at the same time but graphed separately, thus the DMSO control data are the same. Error bars indicate the SD. ${ }^{*} P<6.67 \times 10^{-6} \mathrm{ABT}-737$ and ${ }^{\#} P<6.05$ $\times 10^{-5} \mathrm{ABT}-263$ (ABT vs. DMSO) (C); ${ }^{*} P<2.69 \times 10^{-4} \mathrm{ABT}-737$ and ${ }^{\#} P<3.7$ $\times 10^{-4} \mathrm{ABT}-263$ (vector with $\mathrm{ABT}$ vs. vector, BCL-W, or BCL-2 with DMSO) (D); ${ }^{*} P<7.33 \times 10^{-4}$ ABT-737 and ${ }^{\#} P<0.011$ ABT-263 (BCL-W or BCL-2 with $\mathrm{ABT}$ vs. BCL-W or BCL-2 with DMSO) (E), by $t$ test.

obtained by overexpressing BCL-2 for all experiments with the BH3 mimetics (Figure 8 and Supplemental Figure 14). Collectively, these data show that BLs rely on BCL-W expression and that increased $\mathrm{BCL}-\mathrm{W}$ levels in BL cells confer resistance to the BH3 mimetics ABT-737 and ABT-263.

$B C L-W$ overexpression in diffuse large $B$ cell lymphoma correlates with worse survival. Given our results with $\mathrm{BL}$, we questioned whether BCL-W overexpression was specific to BL or whether BCL-W may contribute to other human B cell lymphomas. We evaluated BCL-W protein levels in diffuse large B cell lymphoma (DLBCL) cell lines, which often have dysregulated MYC activity. Increased BCL-W protein levels were observed in the DLBCL cell lines compared with levels in the normal controls (Figure 6D). Additionally, immunohistochemical analysis of DLBCL patient samples showed that BCL-W protein was expressed in $87.7 \%$ ( $\geq 1$ pathologist score) of the patient samples (Figure 9A, Table 2, Supplemental Figure 15). This frequency was consistent with the frequency $(73.7 \%)$ we observed for elevated BCL-2 levels in DLBCL (Supplemental Figure 15), a known clinical feature of DLBCL (5). Additionally, while many DLBCL patient samples expressed both BCL-W and BCL-2 protein, approximately a quarter of the samples (26.3\%) expressed only BCL-W protein and not BCL-2 protein (Supplemental Figure 15B). Of these samples, the vast majority (80\%) had high (scoring a 2 or 3 ) BCL-W levels.

To determine whether the increase in BCL-W protein in DLBCL may be due to elevated mRNA levels, we performed qRT-PCR on patient samples of DLBCL, with normal lymph nodes and spleens serving as control lymphatic tissue. We determined that $B C L-W$ was significantly overexpressed in DLBCL compared with levels detected in normal tissues (Figure 9B and Supplemental Figure 16). We obtained similar results for $B C L-2$ (Figure 9B and Supplemental Figure 16), which served as a positive control. Furthermore, we evaluated publically available microarray gene expression-profiling data on DLBCL to determine whether our mRNA results were reflected in larger patient populations (http:// www.ncbi.nlm.nih.gov/geo/). Both $B C L-W$ and $B C L-2$ were significantly overexpressed in DLBCL compared with mixed populations of normal B cells or only germinal center B cells (Figure 9C 
A

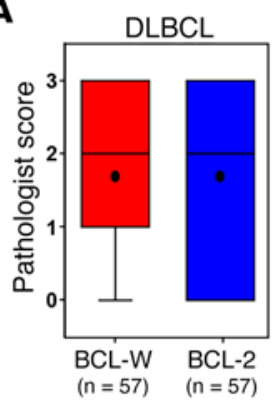

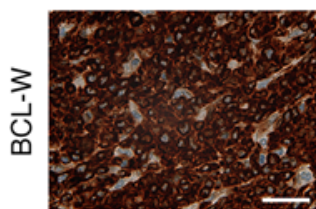
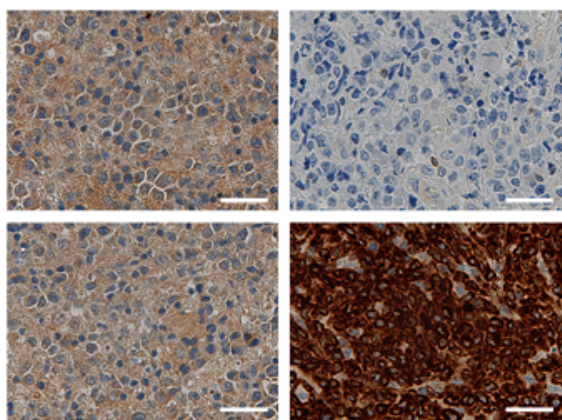
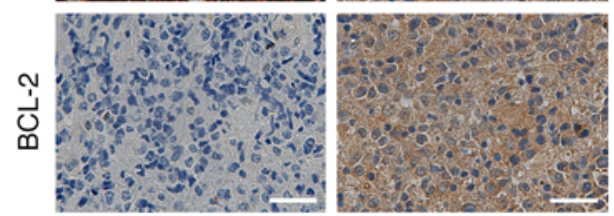

B

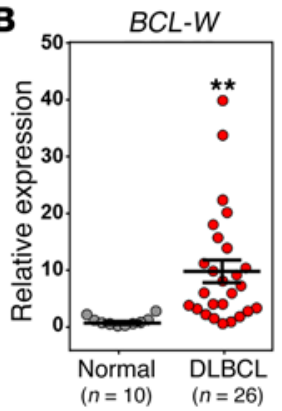

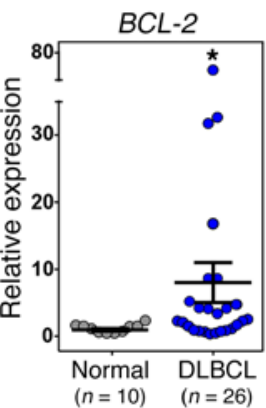
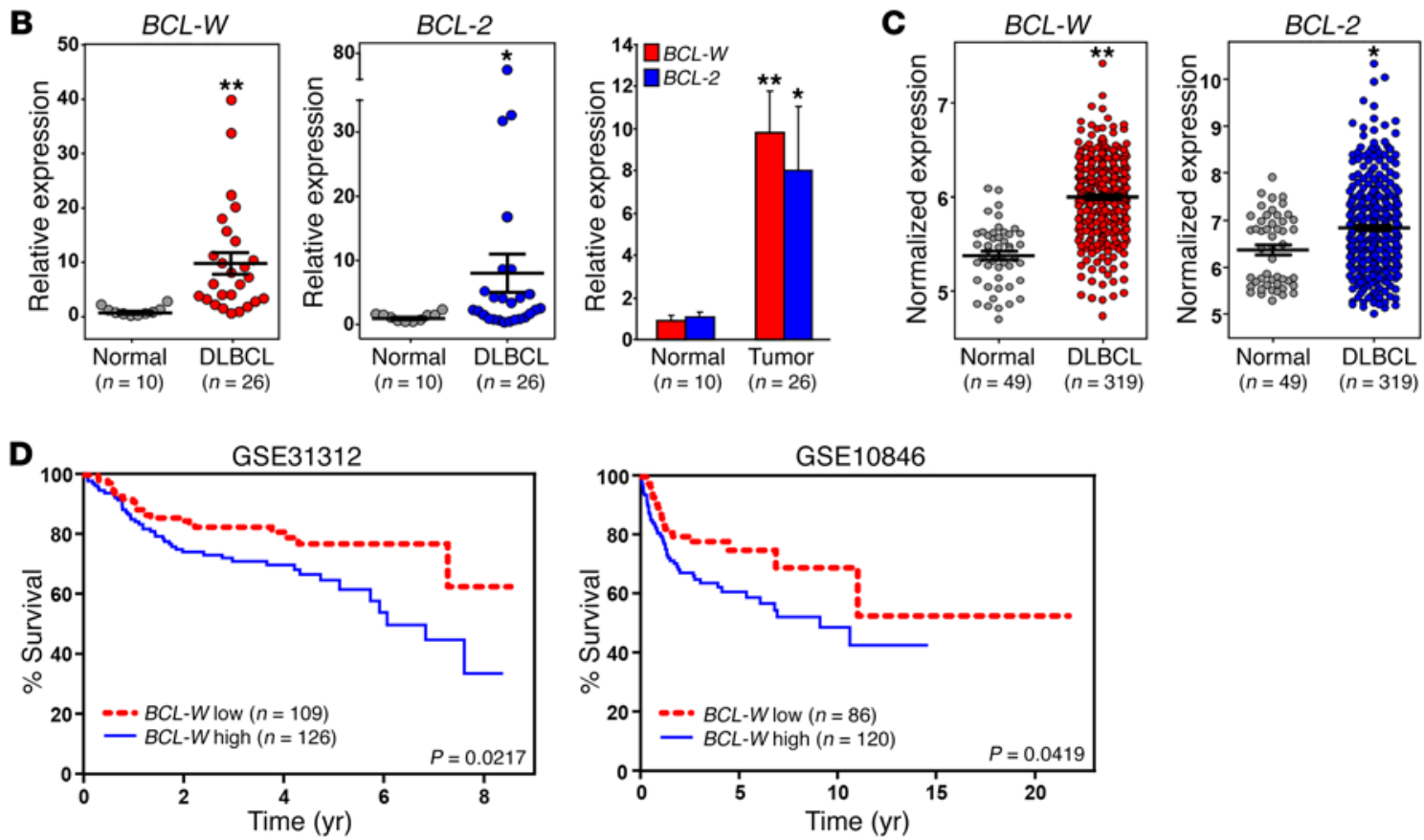

Figure 9. Increased expression of BCL-W in DLBCL correlates with poor patient survival. (A) Immunohistochemical analysis for BCL-W and BCL-2 protein was performed on samples from patients with DLBCL $(n=57)$. Box and whisker plots of pathologist scores and representative images of BCL-W and BCL-2 staining from the same 3 tumor samples. Original magnification, $\times 40$; scale bars: $200 \mu \mathrm{m}$. For the box and whisker plots, the box represents the $25^{\text {th }}$ and $75^{\text {th }}$ percentiles, the line indicates the median, the circle indicates the mean, and the whiskers represent the maximum and minimum. (B) qRT-PCR analysis in triplicate of samples from patients with DLBCL $(n=26)$ compared with normal lymphoid control tissue $(n=10)$. mRNA levels were normalized to $\beta$-actin and are presented as $2^{-\Delta \Delta c t}$. Error bars represent the mean $\pm S E M .{ }^{*} P<6.03 \times 10^{-5}$ and ${ }^{*} P=0.014$, by $t$ test. (C) Microarray gene expressionprofiling data for BCL-W and BCL-2 mRNA in DLBCL $(n=319)$ compared with normal B cells $(n=49)$. Lines represent the mean \pm SEM. Each circle represents 1 sample, and the $y$ axis represents normalized expression of $B C L-W$ and $B C L-2 .{ }^{* *} P<2.2 \times 10^{-16}$ and ${ }^{*} P=9.96 \times 10^{-5}$, by $t$ test. The data sets analyzed are listed in Supplemental Table 1. (D) Patient samples of DLBCL with low BCL-2 from the GSE31312 and GSE10846 data sets were each separated into 2 groups ( $B C L-W$ high and $B C L-W$ low) on the basis of the median expression of $B C L-W$, and Kaplan-Meier analyses were performed. The $P$ values in $\mathbf{D}$ were determined by log-rank test.

and Supplemental Figure 17). Together, our data show that BCL-W may also contribute to DLBCL.

Next, we questioned whether there was a correlation between $B C L-W$ and $B C L-2$ expression in DLBCL. Pearson's correlations were calculated for $B C L-W$ and $B C L-2$ expression for 2 independent data sets. Independently, both data sets showed a negative correlation between $B C L-W$ and $B C L-2$ expression levels in DLBCL patient samples (for GSE10846, $R=-0.1687$ and $P=2.9 \times 10^{-4}$; for GSE31312, $R=-0.0948$ and $P=3.99 \times 10^{-2}$ ). Given that $B C L-W$ was frequently overexpressed in DLBCL and survival rates of DLBCL are not high, we questioned whether increased expression of $B C L-W$ correlated with DLBCL patient survival. To address this, the same 2 DLBCL data sets were evaluated, as both contained survival information. Because $B C L-W$ expression was inversely correlated with $B C L-2$ expression, we assessed the contribution of increased $B C L-W$ expression in samples with lower levels of $B C L-2$. Specifically, patient samples were simultaneously stratified on the basis of the median expression of $B C L-2$ and $B C L-W$ into high or low expression groups for each gene. Then, using data on those patients with low BCL-2 expression, Kaplan-Meier survival curves were plotted for the high and low $B C L-W$ expression groups. For both data sets, the patients with higher $B C L-W$ levels and low $B C L-2$ levels had significantly worse overall survival than did those with lower levels of both $B C L-W$ and $B C L-2(P=0.0217$ for GSE31312 and $P=0.0419$ for GSE10846, log-rank tests, Figure 9D). Stratification of $B C L-W$ expression for patients with high 
Table 3. Analysis of overall survival in patients with DLBCL with low BCL-2 expression

\begin{tabular}{|c|c|c|c|c|c|c|c|}
\hline \multirow[t]{2}{*}{ Variables } & & \multicolumn{3}{|c|}{ Univariate } & \multicolumn{3}{|c|}{ Multivariate } \\
\hline & & HR 95\% & $\mathrm{Cl}$ & $P$ value & HR 95\% & $\mathrm{Cl}$ & $P$ value \\
\hline \multirow[t]{2}{*}{ CSE10846 } & Age & 1.793 & $1.113-2.888$ & 0.015 & 1.898 & $1.166-3.091$ & 0.010 \\
\hline & Tumor stage ${ }^{A}$ & 1.851 & $1.138-3.010$ & 0.012 & 2.057 & $1.255-3.372$ & 0.004 \\
\hline \multirow[t]{2}{*}{ CSE31312 } & Age & 2.252 & $1.366-3.712$ & 0.001 & 2.318 & $1.391-3.861$ & 0.001 \\
\hline & Tumor stage ${ }^{A}$ & 2.388 & $1.444-3.949$ & $4.72 \times 10^{-4}$ & 2.426 & $1.468-4.011$ & $5.5 \times 10^{-4}$ \\
\hline
\end{tabular}

${ }^{A}$ Early stages (I and II) versus late stages (III and IV). HR, hazard ratio.

$B C L-2$ expression levels did not show a statistically significant difference in survival for those who overexpressed both. Next, we performed univariate and multivariate Cox regression analyses on both stratified data sets (with low $B C L-2$ expression), since additional prognostic variables (e.g., patient's age and tumor stage) were available. From the univariate analysis, we determined that $B C L-W$ expression, patient's age, and tumor stage were significantly associated with overall survival (Table 3). Considering all 3 variables, multivariate Cox regression analysis indicated that $B C L-W$ expression independently correlated with overall survival (Table 3). Together, these results indicate that $B C L-W$ expression is a predictive indicator and independent determinant of overall survival in DLBCL patients with lower levels of $B C L-2$.

\section{Discussion}

Prior to the current study, BCL-W was not believed to be an important contributor to hematopoietic cell survival. However, this conclusion was never tested and was based solely on expression levels and on the observation that no overt hematopoietic cell defects were detected in mice lacking $B c l-w$ (6-9). Here, our study has revealed an unexpected and, in our view, significant function for BCL-W in B cell survival and MYC-induced lymphomagenesis. We also provide important mechanistic insight into the regulation of BCL-W expression by MYC in both hematopoietic and nonhematopoietic cells. Most notably, our data provide striking evidence of a survival role for BCL-W in both human BL and DLBCL that we believe will have significant clinical implications.

Antiapoptotic BCL-2 family members inhibit death signals elicited by a number of cellular stressors, including cytokine withdrawal and activation of MYC (2). Given the dogma regarding $\mathrm{BCL}-\mathrm{W}$, we were initially surprised that in B cells, loss of $\mathrm{Bcl}-\mathrm{w}$ conferred sensitivity to IL-7 withdrawal and MYC activation. Using multiple ex vivo and in vitro approaches, we demonstrated that BCL-W significantly contributes to inhibiting MYC-induced apoptosis in both hematopoietic and nonhematopoietic cells. Specifically, in the absence of BCL-W, primary pre-B cells and MEFs were considerably more susceptible to MYC-induced apoptosis than were cells that had WT BCL-W. Previously, it was reported that $\mathrm{B}$ cells lacking other antiapoptotic BCL-2 family members (BCL-2, BCL-XL, or MCL-1) were more sensitive to MYC-induced apoptosis (32-34), whereas loss of proapoptotic BCL-2 family members (BIM, BAX, PUMA, or NOXA) conferred resistance to MYC-induced apoptosis $(17,18,35,36)$. The p53 pathway also mediates apoptosis due to MYC dysregulation $(16,37)$. There was a decreased frequency of $\mathrm{p} 53$ pathway inactivation in the lymphomas that emerged in $\mathrm{E} \mu-M Y C$ mice lacking $B c l-w$, suggesting a decreased selective pressure to inactivate the p53 pathway during tumorigenesis. We previously reported that loss of the proapoptotic BCL-2 family member BAX altered the frequency of p53 inactivation in MYC-driven lymphomas (17). Together, our data highlight a previously unknown function of BCL-W in apoptosis triggered by deregulated MYC expression and suggest that BCL-W may have cross-talk with the p53 pathway.

To our knowledge, no link has yet been established between BCL-W expression and MYC activation. Here, we determined that MYC regulates BCL-W expression and identified the underlying mechanism through which this occurs. Specifically, we demonstrated that MYC suppressed the expression of BCL-W independentlyofitsinteractionwith thetranscriptionalactivator MIZ-1 and of $\mathrm{p} 53$, which has been reported to repress the expression of the related family members $B c l-2$ and $B c l-x l(38,39)$. Furthermore, our data revealed that a transcriptionally competent MYC was required for the reduction in BCL-W expression. However, there was no evidence of direct transcriptional activation by MYC, suggesting that MYC was indirectly regulating the expression of BCL-W. We (here) and others (27-29) have shown that $B c l-w$ is a target of the miR-15 family, which is known to be regulated by MYC (40). In addition, we recently demonstrated that MYC downregulates the expression of BCL-2 and BCL-XL by transcriptionally upregulating the levels of the miR-15 and let-7 families, respectively $(22,23)$. Therefore, we hypothesized that MYC was modulating the expression of BCL-W through the miR-15 family. Using luciferase reporter assays and site-specific small molecules designed to block the miR-15 family from binding the 3'-UTR of $B c l-w$, we provided direct evidence that MYC, through the miR-15 family, modulates BCL-W expression. These data offer mechanistic evidence of a link between MYC, the miR15 family, and BCL-W and, we believe, significantly expand our understanding of MYC-mediated apoptosis. Determining which transcriptional targets of MYC are responsible for the biological consequences of MYC dysregulation and how these are altered during cellular transformation provides important insights into lymphomagenesis. The data presented here, combined with previous findings $(22,23,30,40)$, indicate that MYC transcriptionally activates or represses miR to induce growth or apoptosis and that this is altered in cancers. 
With our data showing that BCL-W is a critical mediator of MYC-induced apoptosis, we hypothesized that loss of $B c l$-w would affect lymphomagenesis driven by dysregulated MYC. While $\mathrm{Bcl}$-w heterozygosity delayed MYC-induced lymphoma development, remarkably, $\mathrm{Bcl}-\mathrm{w}^{-/-} \mathrm{E} \mu-M Y C-\mathrm{Tg}$ mice developed lymphoma with a substantially extended latency compared with that of littermate-matched control E $\mu-M Y C$ mice. Specifically, $\mathrm{E} \mu-M Y C$ mice lacking both $B c l-w$ alleles had a mean lifespan that was more than 3 times longer than that of their $B c l-w^{+/+} \mathrm{E} \mu-M Y C$ siblings. By the time all of the $B c l-w^{+/+} \mathrm{E} \mu-M Y C$ mice had succumbed to lymphoma, none of the $\mathrm{Bcl}-\mathrm{w}^{-/} \mathrm{E} \mu-\mathrm{MYC}$ mice had developed lymphoma. Reports of the effects of deleting other prosurvival BCL-2 family members have shown differing results. For example, a $B c l-x l$ deficiency only slightly delayed E $\mu-M Y C$ lymphoma development (33), whereas deletion of $B c l-2$ did not significantly alter lymphomagenesis (34). In contrast, loss of 1 allele of $\mathrm{Mcl}-1$ profoundly delayed MYC-induced lymphoma development (41). Therefore, although there are multiple antiapoptotic BCL-2 family members, they do not appear to equally contribute to MYCinduced B cell lymphomagenesis.

While lymphoma development was profoundly extended in $B c l-w^{-/-} \mathrm{E} \mu-M Y C$ mice, loss of $B c l-w$ did not impact the type of lymphoma that emerged. The lymphomas that arose, regardless of genotype or time of tumor incidence, were characteristic $\mathrm{E} \mu-M Y C$ pre-B/B cell lymphomas. These observations suggested that the $\mathrm{B}$ cell populations in which transformation occurs to generate lymphomas in the E $\mu-M Y C$ mouse model (15) remained unaltered by loss of $\mathrm{Bcl}-\mathrm{w}$. Indeed, flow cytometric analysis revealed that the $\mathrm{B}$ cell populations in the spleen and BM of precancerous littermates were indistinguishable among the 3 genotypes. Previous reports using a transplant model with fetal liver-derived stem cells from E $\mu-M Y C B c l-2^{-/-}$(34) or E $\mu-M Y C B c l-x l^{-/-}$(33) mice documented reduced immature and mature or pro-B and pre-B cells, respectively, which is consistent with the cell types in which these proteins predominantly function (2). Despite having similar biochemical functions, our data support the concept that specific antiapoptotic BCL-2 family members are more important than other family members in different cell types or stages of differentiation (5). Knowing when and how individual prosurvival BCL-2 proteins are relied on during neoplastic transformation may provide additional strategies for treating MYC-driven lymphoma.

MYC is overexpressed and/or dysregulated in the majority of human cancers (3); therefore, we believe that our results demonstrating an essential role for BCL-W in MYC-induced lymphomagenesis have significant implications for cancer biology and therapy. Importantly, we exposed a previously unknown connection between BCL-W and the MYC-driven human BL, a lymphoma for which no other antiapoptotic BCL-2 family member has been linked. Using a combination of approaches, we showed that increased BCL-W expression is highly selected for in BL. Specifically, $88.5 \%$ of the patient samples expressed BCL-W protein, whereas only $15.4 \%$ expressed BCL-2. These data were supported by analyses of $B C L-W$ and $B C L-2$ mRNA from BL patient samples, which showed that elevated levels of $B C L-W$ mRNA were preferentially selected for, but $B C L-2$ was not. Furthermore, we demonstrate that knocking down $B C L-W$ expression in human $\mathrm{BL}$ cell lines resulted in apoptosis, highlighting the importance of main- taining BCL-W levels in BL for continued cell survival. Additionally, elevated levels of BCL-W alone conferred resistance to BL cell lines to the apoptotic effects of the $\mathrm{BH} 3$ mimetic compounds ABT737 and ABT-263, which target 3 antiapoptotic BCL-2 family members. Previously, it was reported that $B C L-2$ expression correlated with sensitivity to ABT-263 in a panel of human non-Hodgkin lymphoma cell lines (42) and sensitivity to ABT-737 in human chronic lymphocytic leukemia $(43,44)$, small-cell lung cancer cell lines (45), and E $\mu-M Y C$ lymphomas $(46,47)$. In addition, increased levels of other antiapoptotic family members, such as $M C L-1$ and $B C L-X L$, have also been shown to confer resistance to ABT737 (42). Therefore, our data indicate that expression of BCL-W will likely need to be considered and monitored when using $\mathrm{BH} 3$ mimetics for the treatment of lymphomas. Moreover, our data suggest that BCL-W may be useful in the diagnosis of BL and aid in the development of targeted therapies for this malignancy.

We also investigated BCL-W in DLBCL with respect to BCL-2, a known contributor to DLBCL that, when overexpressed, correlates with reduced patient survival $(5,48)$. We determined that many samples from DLBCL patients expressed both BCL-W and BCL-2 protein, but approximately a quarter of the samples only expressed BCL-W protein, and of these, the vast majority had high BCL-W levels. Furthermore, gene expression profiling of large DLBCL data sets also showed an increase in $B C L-W$ mRNA in DLBCL compared with normal $\mathrm{B}$ cells. We also determined that there was a negative correlation between $B C L-W$ and $B C L-2$ levels in DLBCL, indicating that there may be a significant subset of DLBCL cells that preferentially selects for the overexpression of BCL-W rather than BCL-2. Importantly, those patients whose lymphoma did preferentially select for $B C L-W$ overexpression while $B C L-2$ levels remained low had markedly worse survival rates, and in this context, the level of $B C L-W$ was also an independent determinant of overall survival in patients with DLBCL. Of note, there was no additive negative affect on patient survival when both $B C L-2$ and $B C L-W$ were overexpressed, suggesting that lymphoma cell survival is not further enhanced once a certain expression level of antiapoptotic BCL-2 family members is reached. In support of this concept, it has been hypothesized that a mechanism of resistance to the $\mathrm{BH}-3$ mimetics involves the upregulation of other antiapoptotic BCL-2 family members that are not inhibited by the compounds (5). Therefore, our data show that $B C L-W$ expression is an important contributor to and predictive indicator of DLBCL. Thus, BCL-W may serve as a novel biomarker for BL and more aggressive DLBCL. Collectively, our data indicate that BCL-W is an understudied contributor to lymphomas that could be added to the limited list of current predictive biomarkers to improve prognostication and the development of more effective individualized treatment strategies.

\section{Methods}

Mice. C57Bl/6 E $\mu-M Y C$-Tg mice (4) were mated with C57Bl/6 Bcl- $w^{+/-}$ mice (9) provided by Rosalind Segal (Harvard Medical School, Harvard University, Boston, Massachusetts, USA), and their offspring were intercrossed to obtain littermate-matched $\mathrm{Bcl}-\mathrm{w}^{+/+}, \mathrm{Bcl}-\mathrm{w}^{+/-}$, and $B c l-w^{-1-} \mathrm{E} \mu-M Y C-T g$ mice of both sexes. Only E $\mu-M Y C-\mathrm{Tg}-$ positive males were mated to pass on the Tg. For survival studies, mice were sacrificed at humane endpoints, and tumors and tissues were harvested and analyzed. 
Lymphoma phenotype and B cell development analysis. Splenocytes and BM from littermates (prior to lymphoma development for $\mathrm{E} \mu-M Y C-\mathrm{Tg}$ mice) or lymphoma cells were analyzed by flow cytometry following incubation with fluorochrome-linked antibodies as previously reported $(49,50)$.

Patient samples and tissue acquisition. Normal human B cells were purified from leukoreduction filters obtained from the Red Cross using the IMag Human B Lymphocyte Enrichment Set (BD Biosciences). Deidentified fresh spleens were obtained from the Cooperative Human Tissue Network at Vanderbilt University Medical Center (VUMC) (Nashville, Tennessee, USA). For immunohistochemical analysis of DLBCL, formalin-fixed, paraffin-embedded (FFPE) tissues were selected from archival pathology materials on the basis of a documented large B cell diagnosis. Two separate tissue microarrays (TMAs) were constructed using 2-mm diameter cores from 35 separate specimens represented in duplicate and 20 separate specimens represented in triplicate, respectively. In addition, 10 samples with high-grade morphologic features were examined as individual slides. Fifty-seven DLBCL samples were considered evaluable and included in the analyses. For qRT-PCR analysis of DLBCL, TissueScan cDNA arrays containing 26 samples from patients with DLBCL and 10 normal lymphoid tissue controls were obtained from OriGene. For immunohistochemical analysis of BL, slides from 28 deidentified patients diagnosed with BL were obtained from St. Jude Children's Research Hospital (Memphis, Tennessee, USA). Twenty-six BL samples were considered evaluable and included in the analyses. Patient characteristics are listed in Table 2, and details on how the pathologist scored IHC are provided in Supplemental Figures 11 and 15. For qRT-PCR analysis of BL samples, 15 FFPE BL samples and 7 FFPE normal lymphoid control tissues were obtained from Thomas Jefferson University (Philadelphia, Pennsylvania, USA).

IHC. Immunohistochemical analysis was performed on $4-\mu \mathrm{m}$ sections from BM preparations with anti-BCL-W (sc-6172; Santa Cruz Biotechnology Inc.) and anti-BCL-2 (PA0117; Leica Biosystems). A Leica BOND-MAX stainer was used with the Bond Polymer Refine detection system for visualization (Leica Biosystems). Expression levels were scored by a practicing board-certified hematopathologist (ASK) on a $0-3$ point scale in a blinded fashion and scoring only the neoplastic cells. After unblinding, scores for the replicate samples on the TMAs were averaged and a final score obtained.

Vectors. MSCV-MYCER-IRES-GFP, MSCV-MYCAMBIIER-IRESGFP (MYC box II deletion mutant), and MSCV-MYCV394D-ER-IRESGFP (MYC:MIZ-1 interaction mutant) bicistronic retroviral vectors have been previously described $(23,37)$. Luciferase reporter plasmids were constructed by cloning a 60-mer of the 3'-UTR of $\mathrm{Bcl}$-w containing the predicted miR-15 family target sequence into pMIR-REPORT (Invitrogen, Thermo Fisher Scientific). Luciferase reporters containing mutated miR binding sites were similarly generated by cloning a 60-mer with a mutated (base substitutions) target sequence to prevent the miR-15 family from binding the $3^{\prime}-\mathrm{UTR}$ of $\mathrm{Bcl}-\mathrm{w}$ into pMIR-REPORT. Both luciferase plasmids for the $B c l$-2 3 '-UTR have previously been reported (23). For luciferase assays, a reporter plasmid encoding $\beta$-gal was used for transfection normalization (Invitrogen, Thermo Fisher Scientific). For knockdown experiments, nontargeting and $B C L-W$ shRNA sequences were cloned into the pInducer Dox-inducible shRNA system (51). To induce shRNA expression, cells were treated with $1 \mu \mathrm{g} / \mathrm{ml}$ Dox (Sigma-Aldrich) or vehicle control (DMSO). For overexpression and res- cue experiments, retroviral vectors were generated to express $B C L-W$ (MSCV-BCLW-IRES-RFP) or BCL-2 (MSCV-BCL2-IRES-RFP).

Cells, infection, and transfection. Daudi, Ramos, Raji, Su-DHL-6, and NIH3T3 cells were cultured as described by the American Type Culture Collection (ATCC). OCI-Ly-19 and OCI-Ly-3 cells were cultured in RPMI-1640 containing 10\% FBS. All cell lines were negative for MYCoplasma. MEFs were generated and cultured as previously described (37). Primary murine pre-B cell cultures were generated as previously described (16). Briefly, BM was harvested from 8- to 10-week-old $\mathrm{Bcl}-\mathrm{w}^{+/+}, \mathrm{Bcl}-\mathrm{w}^{+/}$, and $\mathrm{Bcl}-\mathrm{w}^{-/-}$littermate-matched mice or 4- to 6-weekold littermate-matched E $\mu-M Y C$-Tg mice (prior to lymphoma development) with 1, 2, or no Bcl-w alleles. After red blood cell lysis, BM cells were cultured in RMPI supplemented with $10 \mathrm{ng} / \mathrm{ml} \mathrm{IL-7,20 \%} \mathrm{FBS,} 55$ $\mu \mathrm{M} \beta$-mercaptoethanol, $2 \mathrm{mM}$ glutamine, and penicillin-streptoMYCin. Primary pre-B cells and MEFs were infected with bicistronic retroviral vectors (described above) in the presence of $8 \mu \mathrm{g} / \mathrm{ml}$ polybrene as previously described (37). GFP-positive pre-B cells were isolated by fluorescence-activated cell sorting. Human BL cell lines were infected with Dox-inducible lentiviral vectors (see above) expressing either a nontargeting shRNA or $B C L-W$-targeting shRNA and/or retroviral vectors expressing either $B C L-W$ or $B C L-2$ cDNA or empty vector control. $p 53^{-/-}$ MEFs were transfected using Lipofectamine 2000 (Invitrogen, Thermo Fisher Scientific) with $200 \mathrm{nM}$ miScript Target Protector for $\mathrm{Bcl}-\mathrm{w}$ or Bcl-2 (QIAGEN) according to the manufacturers' protocols.

Western blotting. Whole-cell protein lysates were generated, and equal amounts of protein per lane were Western blotted as previously described (16). The following antibodies were used: p19ARF (GTX200780; GeneTex); p53 (Ab-7; Calbiochem); BCL-W (31H4, product no. 2724) and cleaved caspase 3 (Asp175, product no. 9661) (both from Cell Signaling Technology); BCL-2 (3F11) and BCL-XL (44/BCL-X) (both from BD Bioscience); MCL-1 (200-401-CR9; Rockland); MYC (9E10; EMD Millipore); and $\beta$-actin (AC-15; SigmaAldrich). p53 cDNA was sequenced as previously described (16).

Growth and apoptosis analyses. For IL-7 deprivation experiments, BM-derived primary pre-B cells were washed twice with PBS and resuspended in complete media (described above) without IL-7. To activate MYCER, $1 \mu \mathrm{M}$ 4-hydroxytamoxifen (4-OHT; Sigma-Aldrich) or vehicle control (EtOH) was added to culture media. Population doublings, cell numbers, and/or viability were determined by trypan blue dye exclusion (in triplicate) and proliferation by MTT (Sigma-Aldrich; $560 \mathrm{~nm}$ ) or MTS (Promega; $490 \mathrm{~nm}$ ) assays (in quadruplicate). Apoptosis was evaluated by Western blotting for cleaved caspase 3 (described above) and flow cytometry following propidium iodide (sub-G1/apoptotic DNA content) or annexin V/7-AAD staining (both in triplicate) as previously reported (52). The ABT-737 and ABT-263 compounds were obtained from Selleckchem and reconstituted with DMSO.

$q R T-P C R$. Total RNA was isolated from cell lines using TRIzol (Invitrogen, Thermo Fisher Scientific) or the RecoverAll Total Nucleic Acid Isolation Kit for FFPE tissue (Thermo Fisher Scientific) according to the manufacturer's protocols. cDNA was generated and SYBR Green (SABiosciences, QIAGEN) and TaqMan MicroRNA (Applied Biosystems) assays were performed in triplicate to measure mRNA and miR, respectively, as previously described $(53,54)$. qPCR of lymphoma TissueScan cDNA arrays (OriGene) was completed according to the manufacturer's protocol. mRNA and miR expression was normalized to $\beta$-actin and $R N U 6 b$, respectively, and presented as $2^{-\Delta \Delta \mathrm{Ct}}$. Primer sequences for mouse and human $B C L-W, B C L-2, B C L-X L$, 
$M C L-1$, and $\beta$-actin mRNA expression were obtained from the Harvard Medical School Primer Bank (55).

Luciferase assays. p53/- MEFs expressing the 4-OHT-inducible WT MYCER, MYCAMBII-ER, or MYCV394D-ER were transfected in triplicate with luciferase reporters (described above), $\beta$-gal control plasmid, and/or $200 \mathrm{nM}$ miScript Target Protectors (QIAGEN) designed to block miR-15 family binding sites in the 3'-UTR of Bcl-w and $B c l$-2. Luciferase and $\beta$-gal activity was measured 24 hours after MYCER activation as previously described (23).

Microarray gene expression analysis. Microarray gene expression profiling data for BL, DLBCL, and normal human B cells (germinal center centroblasts and centrocytes, naive B cells, and memory B cells) were generated on the Affymetrix Human Genome U133 Plus 2.0 platform following the standard Affymetrix protocol. Data sets (summarized in Supplemental Table 1) were downloaded from the NCBI's Gene Expression Omnibus (GEO) database (56) or from the authors' website. BL, DLBCL, and normal B cell CEL files were normalized using a robust multiarray average algorithm (57). Batch effects were adjusted using ComBat (58). Probe sets were averaged to obtain a single-expression intensity measure per gene per array. Differential expression was measured by an unpaired, 1-tailed $t$ test. All analyses were carried out in $\mathrm{R}$, version 3.2.3.

Patient survival and correlation analyses. Two microarray gene expression-profiling data sets for DLBCLs $(59,60)$ were generated on the Affymetrix Human Genome U133 Plus 2.0 platform following the standard Affymetrix protocol. These data sets and clinicopathological information were downloaded from the NCBI's GEO database (GSE10846 and GSE31312). For each data set, raw expressionprofiling data (CEL files) were normalized using a robust multiarray average algorithm (57). Probe sets were averaged to obtain a singleexpression intensity measure per gene per array. For survival analyses, patient samples were simultaneously stratified on the basis of the median expression of $B C L-2$ and $B C L-W$ into high or low expression groups for each gene. Then, using data on those patients with low $B C L-2$ expression, Kaplan-Meier survival curves were plotted for the high and low $B C L-W$ expression groups and compared using log-rank tests. Univariate and multivariate Cox proportional hazards regression analyses for overall DLBCL survival outcomes based on $B C L-W$ expression when $B C L-2$ expression was low were also performed. Tumor stages were stratified into lower (I and II) and higher (III and IV) stages. Patients were divided into lower and higher age groups on the basis of the median age. Survival analyses were carried out using the R survival package, version 3.2.3.

Statistics. A 1-tailed Student's $t$ test was used when comparing 2 groups. One-way ANOVA was performed with Bonferroni's correction when comparing multiple groups. Survival curves were compared by log-rank tests. The figure legends indicate the statistical tests used, which groups were compared, and when values represent the mean \pm $\mathrm{SD}$ or the mean $\pm \mathrm{SEM}$. Univariate and multivariate survival analyses were analyzed by Cox proportional hazards regression models. The Pearson's correlation coefficient was used to determine the association between $B C L-W$ and $B C L-2$ expression. A $P$ value of less than 0.05 was considered statistically significant.

Study approval. All studies using mice and deidentified human samples complied with state and federal guidelines and were approved by the IACUCs and/or IRBs of VUMC and Thomas Jefferson University. All deidentified human tissues were obtained from the Cooperative Human Tissue Network of VUMC, St. Jude Children's Research Hospital, or Thomas Jefferson University.

\section{Author contributions}

CMA and CME designed the research studies. CMA performed the experiments. ASK performed the pathological analyses. RM completed the bioinformatics analyses. JKC and JZG provided samples from patients with $\mathrm{BL}$ for immunohistochemical and mRNA analyses, respectively. CMA, ASK, RM, and CME wrote the manuscript. All authors reviewed and edited the manuscript.

\section{Acknowledgments}

We thank Pia Arrate, Kelli Boyd, and Cindy Lowe for their technical assistance; Rosalind Segal for the Bcl-w mice; the Encyclopedia of DNA Elements (ENCODE) Consortium and production laboratories; and members of the Eischen laboratory for their helpful discussions. TMAs and IHC were performed by the Translational Pathology Shared Resource at VUMC. Support was provided by NCI for F31CA165728 (CMA), R01CA177786 (CME), R01CA148950 (CME), and the NCI Cancer Center support grant P30CA056036.

Address correspondence to: Christine M. Eischen, Thomas Jefferson University, Department of Cancer Biology, 233 South $10^{\text {th }}$ Street, Philadelphia, Pennsylvania 19107, USA. Phone: 215. 503.5692; E-mail: christine.eischen@jefferson.edu.
1. Hanahan D, Weinberg RA. Hallmarks of cancer: the next generation. Cell. 2011;144(5):646-674.

2. Hata AN, Engelman JA, Faber AC. The BCL2 family: Key mediators of the apoptotic response to targeted anticancer therapeutics. Cancer Discov. 2015;5(5):475-487.

3. Stine ZE, Walton ZE, Altman BJ, Hsieh AL, Dang CV. MYC, metabolism, and cancer. Cancer Discov. 2015;5(10):1024-1039.

4. Adams JM, et al. The c-MYC oncogene driven by immunoglobulin enhancers induces lymphoid malignancy in transgenic mice. Nature. 1985;318(6046):533-538.

5. Delbridge AR, Grabow S, Strasser A, Vaux DL. Thirty years of BCL-2: translating cell death discoveries into novel cancer therapies. Nat Rev Cancer. 2016;16(2):99-109.
6. Gibson L, et al. bcl-w, a novel member of the bcl-2 family, promotes cell survival. Oncogene. 1996;13(4):665-675.

7. O'Reilly LA, et al. Tissue expression and subcellular localization of the pro-survival molecule Bcl-w. Cell Death Differ. 2001;8(5):486-494.

8. Print CG, et al. Apoptosis regulator bcl-w is essential for spermatogenesis but appears otherwise redundant. Proc Natl Acad Sci USA. 1998;95(21):12424-12431.

9. Ross AJ, et al. Testicular degeneration in Bclwdeficient mice. Nat Genet. 1998;18(3):251-256.

10. Beverly LJ, Varmus HE. MYC-induced myeloid leukemogenesis is accelerated by all six members of the antiapoptotic BCL family. Oncogene. 2009;28(9):1274-1279.

11. Lee HW, Lee SS, Lee SJ, Um HD. Bcl-w is expressed in a majority of infiltrative gastric adenocarcinomas and suppresses the cancer cell death by blocking stress-activated protein kinase/c-Jun NH2-terminal kinase activation. Cancer Res. 2003;63(5):1093-1100.

12. Wilson JW, et al. Bcl-w expression in colorectal adenocarcinoma. Br JCancer. 2000;82(1):178-185.

13. Placzek WJ, Wei J, Kitada S, Zhai D, Reed JC, Pellecchia M. A survey of the anti-apoptotic Bcl-2 subfamily expression in cancer types provides a platform to predict the efficacy of Bcl-2 antagonists in cancer therapy. Cell Death Dis. 2010;1:e40.

14. Littlewood TD, Hancock DC, Danielian PS, Parker MG, Evan GI. A modified oestrogen receptor ligand-binding domain as an improved switch for the regulation of heterologous proteins. Nucleic Acids Res. 1995;23(10):1686-1690. 
15. Harris AW, Pinkert CA, Crawford M, Langdon WY, Brinster RL, Adams JM. The E mu-MYC transgenic mouse. A model for high-incidence spontaneous lymphoma and leukemia of early B cells. JExp Med. 1988;167(2):353-371.

16. Eischen CM, Weber JD, Roussel MF, Sherr CJ, Cleveland JL. Disruption of the ARF-Mdm2-p53 tumor suppressor pathway in MYC-induced lymphomagenesis. Genes Dev. 1999;13(20):2658-2669.

17. Eischen CM, Roussel MF, Korsmeyer SJ, Cleveland JL. Bax loss impairs MYC-induced apoptosis and circumvents the selection of p53 mutations during MYC-mediated lymphomagenesis. Mol Cell Biol. 2001;21(22):7653-7662.

18. Egle A, Harris AW, Bouillet P, Cory S. Bim is a suppressor of MYC-induced mouse B cell leukemia. Proc Natl Acad Sci USA. 2004;101(16):6164-6169.

19. Muller PA, Vousden KH. Mutant p53 in cancer: new functions and therapeutic opportunities. Cancer Cell. 2014;25(3):304-317.

20. Eischen CM, Woo D, Roussel MF, Cleveland JL. Apoptosis triggered by MYC-induced suppression of $\mathrm{Bcl}-\mathrm{X}(\mathrm{L})$ or Bcl-2 is bypassed during lymphomagenesis. Mol Cell Biol. 2001;21(15):5063-5070.

21. Eischen $\mathrm{CM}$, et al. Bcl-2 is an apoptotic target suppressed by both c-MYC and E2F-1. Oncogene. 2001;20(48):6983-6993.

22. Adams CM, Eischen CM. Histone deacetylase inhibition reveals a tumor-suppressive function of MYC-regulated miRNA in breast and lung carcinoma. Cell Death Differ. 2016;23(8):1312-1321.

23. Adams CM, Hiebert SW, Eischen CM. MYC induces miRNA-mediated apoptosis in response to HDAC inhibition in hematologic malignancies. Cancer Res. 2016;76(3):736-748.

24. Eilers M, Eisenman RN. MYC's broad reach. Genes Dev. 2008;22(20):2755-2766.

25. Herold S, et al. Negative regulation of the mammalian UV response by MYC through association with Miz-1. Mol Cell. 2002;10(3):509-521.

26. ENCODE Project Consortium. An integrated encyclopedia of DNA elements in the human genome. Nature. 2012;489(7414):57-74.

27. Yang X, et al. miRNA-195 sensitizes human hepatocellular carcinoma cells to 5-FU by targeting BCL-w. Oncol Rep. 2012;27(1):250-257.

28. Yin KJ, et al. miR-497 regulates neuronal death in mouse brain after transient focal cerebral ischemia. Neurobiol Dis. 2010;38(1):17-26.

29. Yang T, et al. MicroRNA-15a induces cell apoptosis and inhibits metastasis by targeting BCL2L2 in non-small cell lung cancer. Tumour Biol. 2015;36(6):4357-4365.

30. Chang TC, et al. Widespread microRNA repression by MYC contributes to tumorigenesis. Nat Genet. 2008;40(1):43-50.

31. Schmitz R, Ceribelli M, Pittaluga S, Wright G, Staudt LM. Oncogenic mechanisms in Burkitt lymphoma. Cold Spring Harb Perspect Med. 2014;4(2): a014282.

32. Kelly GL, et al. Targeting of MCL-1 kills MYC-driven mouse and human lymphomas even when they bear mutations in $\mathrm{p} 53$. Genes Dev. 2014;28(1):58-70.

33. Kelly PN, Grabow S, Delbridge AR, Strasser A, Adams JM. Endogenous Bcl-xL is essential for MYC-driven lymphomagenesis in mice. Blood. 2011;118(24):6380-6386.

34. Kelly PN, Puthalakath H, Adams JM, Strasser A. Endogenous bcl-2 is not required for the development of Emu-MYC-induced B-cell lymphoma. Blood. 2007;109(11):4907-4913.

35. Michalak EM, et al. Puma and to a lesser extent Noxa are suppressors of MYC-induced lymphomagenesis. Cell Death Differ. 2009;16(5):684-696.

36. Garrison SP, et al. Selection against PUMA gene expression in MYC-driven B-cell lymphomagenesis. Mol Cell Biol. 2008;28(17):5391-5402.

37. Zindy $\mathrm{F}$, et al. MYC signaling via the ARF tumor suppressor regulates p53-dependent apoptosis and immortalization. Genes Dev. 1998;12(15):2424-2433.

38. Haldar S, Negrini M, Monne M, Sabbioni S, Croce CM. Down-regulation of bcl- 2 by p 53 in breast cancer cells. Cancer Res. 1994;54(8):2095-2097.

39. Sugars KL, Budhram-Mahadeo V, Packham G, Latchman DS. A minimal Bcl-x promoter is activated by Brn-3a and repressed by p53. Nucleic Acids Res. 2001;29(22):4530-4540.

40. Bui TV, Mendell JT. MYC: Maestro of microRNAs. Genes Cancer. 2010;1(6):568-575.

41. Grabow S, Delbridge AR, Aubrey BJ, Vandenberg CJ, Strasser A. Loss of a single Mcl-1 allele inhibits MYC-driven lymphomagenesis by sensitizing pro-B cells to apoptosis. Cell Rep. 2016;14(10):2337-2347.

42. Mérino D, et al. Bcl-2, Bcl-x(L), and Bcl-w are not equivalent targets of ABT-737 and navitoclax (ABT-263) in lymphoid and leukemic cells. Blood 2012;119(24):5807-5816.

43. Del Gaizo Moore V, Brown JR, Certo M, Love TM, Novina CD, Letai A. Chronic lymphocytic leukemia requires BCL2 to sequester prodeath BIM, explaining sensitivity to BCL2 antagonist ABT-737. J Clin Invest. 2007;117(1):112-121.

44. Al-Harbi S, et al. An antiapoptotic BCL-2 family expression index predicts the response of chronic lymphocytic leukemia to ABT-737. Blood. 2011;118(13):3579-3590.

45. Tahir SK, et al. Influence of Bcl-2 family members on the cellular response of small-cell lung cancer cell lines to ABT-737. Cancer Res. 2007;67(3):1176-1183.

46. Whitecross KF, et al. Defining the target specificity of ABT-737 and synergistic antitumor activities in combination with histone deacetylase inhibi- tors. Blood. 2009;113(9):1982-1991.

47. Mason KD, et al. In vivo efficacy of the Bcl-2 antagonist ABT-737 against aggressive MYC-driven lymphomas. Proc Natl Acad Sci USA. 2008;105(46):17961-17966.

48. Thieblemont C, Brière J. MYC, BCL2, BCL6 in DLBCL: impact for clinics in the future? Blood. 2013;121(12):2165-2166.

49. Arrate MP, Vincent T, Odvody J, Kar R, Jones SN, Eischen CM. MicroRNA biogenesis is required for MYC-induced B-cell lymphoma development and survival. Cancer Res. 2010;70(14):6083-6092.

50. Alt JR, Greiner TC, Cleveland JL, Eischen CM. $\mathrm{Mdm} 2$ haplo-insufficiency profoundly inhibits MYC-induced lymphomagenesis. EMBO J. 2003;22(6):1442-1450.

51. Meerbrey KL, et al. The pINDUCER lentiviral toolkit for inducible RNA interference in vitro and in vivo. Proc Natl Acad Sci USA. 2011;108(9):3665-3670.

52. Adams CM, Eischen CM. Inactivation of p53 is insufficient to allow B cells and B-cell lymphomas to survive without Dicer. Cancer Res. 2014;74(14):3923-3934.

53. Wang P, Lushnikova T, Odvody J, Greiner TC, Jones SN, Eischen CM. Elevated Mdm2 expression induces chromosomal instability and confers a survival and growth advantage to B cells. Oncogene. 2008;27(11):1590-1598.

54. McGirt LY, Adams CM, Baerenwald DA, Zwerner JP, Zic JA, Eischen CM. miR-223 regulates cell growth and targets proto-oncogenes in MYCosis fungoides/cutaneous T-cell lymphoma. J Invest Dermatol.2014;134(4):1101-1107.

55. Wang X, Spandidos A, Wang H, Seed B. PrimerBank: a PCR primer database for quantitative gene expression analysis, 2012 update. Nucleic Acids Res. 2012;40(Database issue):D1144-D1149.

56. Barrett T, Edgar R. Gene expression omnibus: microarray data storage, submission, retrieval, and analysis. Meth Enzymol. 2006;411:352-369.

57. Irizarry RA, et al. Exploration, normalization, and summaries of high density oligonucleotide array probe level data. Biostatistics. 2003;4(2):249-264.

58. Johnson WE, Li C, Rabinovic A. Adjusting batch effects in microarray expression data using empirical Bayes methods. Biostatistics. 2007;8(1):118-127.

59. Lenz $\mathrm{G}$, et al. Stromal gene signatures in large-B-cell lymphomas. $N$ EnglJMed. 2008;359(22):2313-2323.

60. Visco C, et al. Comprehensive gene expression profiling and immunohistochemical studies support application of immunophenotypic algorithm for molecular subtype classification in diffuse large B cell lymphoma: a report from the International DLBCL Rituximab-CHOP Consortium Program Study. Leukemia. 2012;26(9):2103-2113. 